\title{
On biorthogonal systems whose functionals are finitely supported
}

\author{
by \\ Christina Brech (Campinas and São Paulo) and \\ Piotr Koszmider (Łódź and Warszawa)
}

\begin{abstract}
We show that for each natural number $n>1$, it is consistent that there is a compact Hausdorff totally disconnected space $K_{2 n}$ such that $C\left(K_{2 n}\right)$ has no uncountable (semi)biorthogonal sequence $\left(f_{\xi}, \mu_{\xi}\right)_{\xi \in \omega_{1}}$ where $\mu_{\xi}$ 's are atomic measures with supports consisting of at most $2 n-1$ points of $K_{2 n}$, but has biorthogonal systems $\left(f_{\xi}, \mu_{\xi}\right)_{\xi \in \omega_{1}}$ where $\mu_{\xi}$ 's are atomic measures with supports consisting of $2 n$ points. This complements a result of Todorcevic which implies that it is consistent that such spaces do not exist: he proves that its is consistent that for any nonmetrizable compact Hausdorff totally disconnected space $K$, the Banach space $C(K)$ has an uncountable biorthogonal system where the functionals are measures of the form $\delta_{x_{\xi}}-\delta_{y_{\xi}}$ for $\xi<\omega_{1}$ and $x_{\xi}, y_{\xi} \in K$. It also follows from our results that it is consistent that the irredundance of the Boolean algebra $\operatorname{Clop}(K)$ for a totally disconnected $K$ or of the Banach algebra $C(K)$ can be strictly smaller than the sizes of biorthogonal systems in $C(K)$. The compact spaces exhibit an interesting behaviour with respect to known cardinal functions: the hereditary density of the powers $K_{2 n}^{k}$ is countable up to $k=n$ and it is uncountable (even the spread is uncountable) for $k>n$.
\end{abstract}

1. Introduction. If $X$ is a Banach space and $X^{*}$ is its dual, then $\left(x_{i}, x_{i}^{*}\right)_{i \in I} \subseteq X \times X^{*}$ is called a biorthogonal system if $x_{i}^{*}\left(x_{i}\right)=1$ and $x_{i}^{*}\left(x_{j}\right)=0$ if $i \neq j$ for each $i, j \in I$. If $\alpha$ is an ordinal, a transfinite sequence $\left(x_{i}, x_{i}^{*}\right)_{i<\alpha} \subseteq X \times X^{*}$ is called a semibiorthogonal sequence whenever $x_{i}^{*}\left(x_{i}\right)=1, x_{i}^{*}\left(x_{j}\right)=0$ for $j<i<\alpha$ and $x_{i}^{*}\left(x_{j}\right) \geq 0$ for $i<j<\alpha$.

Biorthogonal systems have always played an important role in the theory of Banach spaces ([9]) because all kinds of bases in Banach spaces are in particular the $X$-parts of biorthogonal systems ([20] and [21]). Semibiorthogonal sequences have been introduced quite recently ([2]) in connection with subsets of Banach spaces supported by all of their points ([17], [13], [8]).

2010 Mathematics Subject Classification: Primary 46B26; Secondary 03E35, 54D80.

Key words and phrases: biorthogonal system, $C(K)$ space, irredundant set, unordered $N$-split Cantor set. 
We will mainly deal with biorthogonal systems in real Banach spaces $C(K)$ of all real-valued continuous functions on a compact Hausdorff space $K$ with the supremum norm. Its dual space is isometric to the Banach space $M(K)$ of all Radon measures on $K$ with the variation norm, and so we will identify this dual with $M(K)$. If $K$ is a compact Hausdorff space and $x \in K$, then $\delta_{x}$ denotes the functional on $C(K)$ defined by $\delta_{x}(f)=f(x)$ for all $f \in C(K)$.

This paper is motivated by the following question: If there is an uncountable biorthogonal system $\left(f_{\xi}, \mu_{\xi}\right)_{\xi \in \omega_{1}}$ in $C(K) \times M(K)$, is there also one such that

$$
\mu_{\xi}=\delta_{x_{\xi}}-\delta_{y_{\xi}}
$$

for some points $x_{\xi}, y_{\xi} \in K$ ? Following [4], we will call such biorthogonal systems nice.

The origin of this question is that in all concrete situations so far analyzed in the literature, the above question has a positive answer. Moreover, it happens for a good reason, namely, it follows from a recent result of Todorcevic that Martin's axiom together with the negation of the continuum hypothesis implies a positive answer for $K$ totally disconnected. Indeed, analyzing the proof of Theorem 11 of [24], one gets two cases: the first case, when $K$ is hereditarily separable, which is the main part of that proof and where the constructed biorthogonal system is nice; and the second case, when $K$ is c.c.c. but contains a nonseparable subspace, in which case the proof of Theorem 10 of [24] provides the required nice system; if $K$ is not c.c.c., one can easily obtain an uncountable nice biorthogonal system.

There is one more reason why nice biorthogonal systems appear frequently in the context of Banach spaces $C(K)$ and which makes them more meaningful. Namely, a family $\left(f_{\alpha}\right)_{\alpha \in \kappa}$ is the $X$-part of a nice biorthogonal system if and only if $\left(f_{\alpha}\right)_{\alpha \in \kappa}$ is irredundant in the Banach algebra $C(K)$, in the sense that no $f_{\alpha}$ belongs to the Banach subalgebra generated by the remaining elements. This is a consequence of the Stone-Weierstrass theorem. If $K$ is totally disconnected and $f_{\alpha}$ 's are the characteristic functions of clopen sets $A_{\alpha} \subseteq K$, we obtain the well-known notion of an irredundant set in a Boolean algebra, i.e., a set where no element belongs to the Boolean algebra generated by the remaining elements (see [14]). The irredundance of a Boolean algebra is the supremum of the cardinalities of irredundant sets.

To formulate our main results properly we need the following:

Definition 1.1. Let $K$ be a compact Hausdorff space and $n \in \mathbb{N}$. We say that the functionals of a sequence $\left(f_{\xi}, \mu_{\xi}\right)_{\xi \in \omega_{1}} \subseteq C(K) \times M(K)$ are $n$-supported if each $\mu_{\xi}$ is an atomic measure whose support consists of no more than $n$ points of $K$. 
THEOREM 1.2. For each natural number $n>1$, it is consistent that there is a compact Hausdorff totally disconnected space $K_{2 n}$ such that $C\left(K_{2 n}\right)$ has no uncountable semibiorthogonal sequence whose functionals are $2 n-1$ supported, but has uncountable biorthogonal systems whose functionals are $2 n$-supported.

Moreover, $K_{2 n}^{n}$ is hereditarily separable but $K_{2 n}^{n+1}$ has an uncountable discrete subspace. Neither the Banach algebra $C\left(K_{2 n}\right)$ nor the Boolean algebra $\operatorname{Clop}\left(K_{2 n}\right)$ have an uncountable irredundant family. In particular, $C\left(K_{4}\right)$ has an uncountable biorthogonal system but it has no uncountable nice biorthogonal system.

This situation suggests many questions about the size of biorthogonal systems of various types in $C(K)$ spaces as well as in general Banach spaces. These more general discussions will appear elsewhere. In particular, we are unable to obtain $K$ 's such that $C(K)$ contains biorthogonal systems whose functionals are $2 n+1$-supported but does not contain one whose functionals are $2 n$-supported. The reason why some fundamental change in the approach would have to be taken to obtain such a space is shown in Lemma 3.9 .

On the other hand, if $n=1$ one has absolute results. If $K$ is the split interval, then $K$ is hereditarily separable, and so it cannot have an uncountable semibiorthogonal system whose functionals are 1-supported, but $C(K)$ has an uncountable nice biorthogonal system (see [7]).

It seems that our compact space is the first example showing that the hereditary density or spread of finite powers of a compact space may change its value from countable to uncountable arbitrarily high in $\mathbb{N}$. Such an example can be only consistent since, for example, under MA $+\neg \mathrm{CH}$ if $K^{3}$ is hereditarily separable for a compact $K$, then it is metrizable, and so all finite powers are hereditarily separable. This follows from the fact that under these assumptions there are no compact $S$-spaces ([22]), from the Katětov theorem ([10]) and from the fact that Lindelöf regular spaces are normal.

The paper is organized as follows: in the next section we discuss a general form of the compact spaces we construct and call them unordered $N$-split Cantor sets. They are versions of the split interval whose connection with biorthogonal systems in Banach spaces was already demonstrated in [7]. Section 3 is devoted to a generic construction of Boolean algebras whose Stone spaces are the $K_{2 n}$ 's. That is the only section that requires the knowledge of forcing. The partial order we use is a new modification of that of [11, which produced nonseparable $C(K)$ 's with no uncountable semibiorthogonal sequences. Thus our spaces are quite controllable members of the class of compact spaces constructed in [1], [18], [19], [11. In that section we also prove the existence of an uncountable discrete subspace of $K_{2 n}^{n+1}$ and an uncountable biorthogonal system in $C(K)$ whose functionals are $2 n$-supported. 
The section ends with Theorem 3.8, which expresses the random character of the compact space constructed. Later on we use this theorem to prove further properties of that space. Hence, a reader not familiar with forcing may use this theorem for other purposes and read only the following section. The last, fourth section is devoted to applications of Theorem 3.8 to prove that $K_{2 n}^{n}$ is hereditarily separable and that $C\left(K_{2 n}\right)$ has no uncountable semibiorthogonal sequences whose functionals are $2 n-1$-supported.

We use standard notation: for a positive natural number $n$, we put $[n]=$ $\{1, \ldots, n\}$ and $n=\{0, \ldots, n-1\} . A^{B}$ denotes the set of all functions from $B$ into $A$, and so if $2=\{0,1\}$, then $2^{\omega}$ denotes all infinite sequences with terms in $\{0,1\}$, while $2^{n}$ stands for functions from $n$ into $\{0,1\}$; also, $2^{<\omega}=$ $\bigcup\left\{2^{n}: n \in \mathbb{N}\right\}$ and $\langle s\rangle=\left\{x \in 2^{\omega}: s \subseteq x\right\}$ for $s \in 2^{n}$ and some $n \in \mathbb{N}$. If $A, B$ are sets of ordinals, then $A<B$ means that $\alpha<\beta$ for any $\alpha \in A$ and any $\beta \in B$.

2. Unordered $N$-split Cantor sets. Fix a sequence $\mathcal{X}=\left\{x_{\xi}: \xi<\omega_{1}\right\}$ $\subseteq 2^{\omega}$ of distinct elements and $N \in \mathbb{N}$. Let

$$
K_{N}=\left(2^{\omega} \backslash \mathcal{X}\right) \cup(\mathcal{X} \times[N])
$$

and define

$$
V_{s}=\left(\langle s\rangle \cap\left(2^{\omega} \backslash \mathcal{X}\right)\right) \cup((\langle s\rangle \cap \mathcal{X}) \times[N]) .
$$

Definition 2.1. A family $\left(A_{\xi, i}: \xi<\omega_{1}, i \in[N]\right)$ of subsets of $K_{N}$ is called an $N$-splitting family if it satisfies the following conditions:

(1) $\left(x_{\xi}, i\right) \in A_{\xi, i} \subseteq K_{N}$ for each $\xi<\omega_{1}$ and $i \in[N]$;

(2) for each $\xi<\omega_{1}$ the sets $A_{\xi, i}$ are pairwise disjoint;

(3) for each $\xi<\omega_{1}$ we have $K_{N}=A_{\xi, 1} \cup \cdots \cup A_{\xi, N}$;

(4) if $\eta<\xi$, then there are $k \in \mathbb{N}$ and $j \in[N]$ such that $A_{\eta, i} \cap V_{x_{\eta} \mid k} \subseteq$ $A_{\xi, j} \cap V_{x_{\eta} \mid k}$

(5) if $\eta>\xi$ and $x=x_{\eta}$ or $x \in 2^{\omega} \backslash \mathcal{X}$, then there are $k \in \mathbb{N}$ and $j \in[N]$ such that $V_{x \mid k} \subseteq A_{\xi, j}$.

Definition 2.2. Given an $N$-splitting family $\left(A_{\xi, i}: \xi<\omega_{1}, i \in[N]\right)$, we call the space $\left(K_{N}, \mathcal{T}\right)$ an unordered $N$-split Cantor set if the topology $\mathcal{T}$ on $K_{N}$ is defined by indicating neighbourhood bases $\mathcal{B}_{x}$ at $x$ for every $x \in K_{N}$ in the following way: if $x \in 2^{\omega} \backslash \mathcal{X}$, then

$$
\mathcal{B}_{x}=\left\{V_{s}: s \subseteq x\right\},
$$

and if $x=\left(x_{\xi}, j\right) \in K_{N}$, then

$$
\mathcal{B}_{x}=\left\{V_{s} \cap A_{\xi, j}: s \subseteq x_{\xi}\right\} .
$$

The intuitive meaning of the above definitions is the following: each point $x_{\xi}$ of $2^{\omega}$ is split into $N$ points $\left(x_{\xi}, 1\right), \ldots,\left(x_{\xi}, N\right)$. If we view $K_{N}$ as constructed inductively, when at step $\xi<\omega_{1}$ we construct the splitting clopen 
neighbourhoods $A_{\xi, 1}, \ldots, A_{\xi, N}$ of the points $\left(x_{\xi}, 1\right), \ldots,\left(x_{\xi}, N\right)$ and these neighbourhoods split only $x_{\xi}$ and no other previously constructed $\left(x_{\eta}, i\right)$ for $\eta<\xi$ (condition 2.1(4)) nor $x_{\eta}$ for $\eta>\xi$ nor $x \in 2^{\omega} \backslash \mathcal{X}$ (condition 2.1(5)). On the other hand, note that $A_{\xi, i}$ 's may split $x_{\eta}$ for $\eta<\xi$, and in this case, by condition 2.1(4), they do it "the same way" as the $A_{\eta, j}$ 's.

Proposition 2.3. Let $N \in \mathbb{N}$. If $\left(A_{\xi, i}: \xi<\omega_{1}, i \in[N]\right)$ is an $N$ splitting family, then the corresponding unordered $N$-split Cantor set is a compact, Hausdorff, totally disconnected topological space.

Proof. Since $V_{\emptyset}=K_{N}$, conditions (1)-(3) of Definition 2.1 imply that $A_{\xi, i}$ 's are clopen sets. Now using Proposition 1.2.3 of [5], we will prove that the above families satisfy the axioms BP1-BP3 for neighbourhood bases from [5]. The only nontrivial part is to prove that given $x \in V \in \mathcal{B}_{y}$, there is $U \in \mathcal{B}_{x}$ such that $x \in U \subseteq V$.

Suppose $x \in 2^{\omega} \backslash \mathcal{X}$ and $x \in V_{s} \in \mathcal{B}_{y}$. Then $s \subseteq x$ and so $V_{s}$ itself is in $\mathcal{B}_{x}$. If $x \in V_{s} \cap A_{\xi, i}$, we also have $s \subseteq x$ and by (5) of Definition 2.1 there is $k \in \mathbb{N}$ such that $V_{x \mid k} \subseteq A_{\xi, j}$ for some $j \in N$. Put $t=s \cup x \mid k$ and note that $V_{t} \subseteq A_{\xi, j}$, so by disjointness (condition 2.1(2)) we have $j=i$ with $x \in V_{t} \in \mathcal{B}_{x}$ and $V_{t} \subseteq V_{s} \cap A_{\xi, i}$.

Now suppose that $x=\left(x_{\eta}, i\right)$ and $x \in V_{s} \in \mathcal{B}_{y}$, hence $s \subseteq x$ and so $V_{s} \cap A_{\eta, i} \in \mathcal{B}_{x}$ and $x \in V_{s} \cap A_{\eta, i} \subseteq V_{s}$.

Finally, let $x=\left(x_{\eta}, i\right)$ and $x \in V_{s} \cap A_{\xi, j} \in \mathcal{B}_{\left(x_{\xi}, j\right)}$, then $s \subseteq x_{\eta}$.

First consider $\eta<\xi$. Then by (5) of Definition 2.1 there are $k \in \mathbb{N}$ and $j^{\prime}$ such that $A_{\eta, i} \cap V_{x_{\eta} \mid k} \subseteq A_{\xi, j^{\prime}} \cap V_{x_{\eta} \mid k}$ and by disjointness we get $j^{\prime}=j$. So, if we put $t=s \cup x_{\eta} \mid k$, then $A_{\eta, i} \cap V_{t} \subseteq A_{\xi, j} \cap V_{t} \subseteq A_{\xi, j} \cap V_{s}$ and of course $A_{\eta, i} \cap V_{t} \in \mathcal{B}_{\left(x_{\eta}, i\right)}$.

Secondly, if $\eta \geq \xi$ and $\left(x_{\eta}, i\right) \in V_{s} \cap A_{\xi, j}$, we also have $s \subseteq x_{\eta}$ and by 2.1(4) there are $k \in \mathbb{N}$ and $j^{\prime}$ such that $V_{x_{\eta} \mid k} \subseteq A_{\xi, j^{\prime}}$ for some $j^{\prime}$. By disjointness we have $j=j^{\prime}$. If $t=s \cup x_{\eta} \mid k$ we have $V_{t} \subseteq A_{\xi, j}$, so $x \in V_{t} \in \mathcal{B}_{x}$ and $V_{t} \subseteq V_{s} \cap A_{\xi, i}$. This completes the proof that $\mathcal{B}_{x}$ 's form a local neighbourhood base.

The Hausdorff property is easy since basic sets are clopen.

To prove the compactness, suppose $\mathcal{U}$ is an open cover of $K_{N}$. We may assume that it consists of basic open sets. For each $x \in 2^{\omega} \backslash \mathcal{X}$ define $s_{x} \in 2^{<\omega}$ such that $x \in V_{s_{x}} \subseteq U \in \mathcal{U}$ for some $U$, and for each $\xi<\omega_{1}$ define $s_{\xi} \in 2^{<\omega}$ such that $\left(x_{\xi}, i\right) \in V_{s_{\xi}} \cap A_{\xi, i} \subseteq U \in \mathcal{U}$ for some $U$, and for each $1 \leq i \leq N$. This actually implies by (3) of Definition 2.1 that $V_{s_{\xi}}$ is covered by finitely many $U \in \mathcal{U}$.

Now $\left\{\left\langle s_{x}\right\rangle,\left\langle s_{\xi}\right\rangle: x \in 2^{\omega} \backslash \mathcal{X}, \xi<\omega_{1}\right\}$ forms an open cover of $2^{\omega}$ which is compact and so it has a finite subcover, which easily yields a finite subcover of $\mathcal{U}$. 
Definition 2.4. Suppose $N \in \mathbb{N}$ and $K_{N}$ is an unordered $N$-split Cantor set. Under the above notation, we define the following:

- $R_{\xi}=\left\{\left(x_{\xi}, 1\right), \ldots,\left(x_{\xi}, N\right)\right\}$.

- $\mathcal{A}_{\alpha}$ is the subalgebra of $\operatorname{Clop}\left(K_{N}\right)$ generated by $\left(V_{s}: s \in 2^{<\omega}\right)$ and $\left\{A_{\xi, i}: \xi<\alpha, i \in[N]\right\}$ for $\alpha \leq \omega_{1}$.

- $C_{\alpha}$ is the closure (in the norm) of the set of finite linear combinations of characteristic functions of elements of $\mathcal{A}_{\alpha}$ inside $C(K)$.

Note that $C_{0}$ can be naturally identified with $C\left(2^{\omega}\right)$ inside $C(K)$.

Lemma 2.5. Let $N \in \mathbb{N}$ and let $K_{N}$ be an unordered $N$-split Cantor set. For every $n \in \mathbb{N}$ and for every $\alpha \in \omega_{1}$ and every $i \in[N]$ we have

$$
A_{\alpha, i} \backslash V_{x_{\alpha} \mid n} \in \mathcal{A}_{\alpha} .
$$

Proof. By the properties 2.1(4)\&(5) of $A_{\xi, i}$ 's any point of $K_{N} \backslash R_{\alpha}$ has a neighbourhood $V$ such that for every $i \in[N]$ it is included in $A_{\alpha, i}$ or disjoint from $A_{\alpha, i}$ and moreover $V \in \mathcal{A}_{\alpha}$.

Since $A_{\alpha, i} \backslash V_{x_{\alpha} \mid n}$ is a compact subspace of $K_{N} \backslash R_{\alpha}$, we have a finite subcover consisting of subsets, i.e. $A_{\alpha, i} \backslash V_{x_{\alpha} \mid n}$ is the supremum of a finite family of elements of $\mathcal{A}_{\alpha}$ as required.

Let us see the general form of continuous rational simple functions on an unordered $N$-split Cantor set. By a rational simple function we mean a function assuming only finitely many rational values.

Lemma 2.6. Suppose that $N \in \mathbb{N}$ and that $K_{N}$ is an unordered $N$-split Cantor set, $\varepsilon>0, \mu$ is a (regular) Radon measure on $K_{N}$ and $f$ is a continuous rational simple function on $K_{N}$. Then there is a simple rational function $g \in C\left(2^{\omega}\right)$, distinct $\xi_{1}, \ldots, \xi_{k}<\omega_{1}$ and rationals $q_{i, l}$, non-negative integers $m_{i}$ and $s_{i} \in 2^{m_{i}}$ with $s_{i}=x_{\xi_{i}} \mid m_{i}$, for $1 \leq i \leq k \in \omega$ and $1 \leq l<N$, such that

$$
f=g+\sum_{1 \leq i \leq k} \sum_{1 \leq l<N} q_{i, l} \chi_{A_{\xi_{i, l}} \cap V_{s_{i}}}
$$

and

$$
\sum_{1 \leq i \leq k} \max _{1 \leq l<N}\left(\left|q_{i, l}\right|\right)|\mu|\left(V_{s_{i}} \backslash R_{\xi_{i}}\right) \leq \varepsilon .
$$

Proof. By induction on $\xi$ we prove that any continuous simple rational function in $C_{\xi}$ can be written in the form as in the lemma. The StoneWeierstrass theorem and the uncountable cofinality of $\omega_{1}$ imply that the union of $C_{\xi}$ 's is the entire $C\left(K_{N}\right)$.

The limit stage is trivial. So, suppose we have proved the conclusion for $C_{\xi}$ and we are given a continuous simple rational function $f$ in $C_{\xi+1}$. Note that

$$
\bigcap_{m \in \mathbb{N}} V_{x_{\xi} \mid m}=R_{\xi}
$$


Hence, the regularity of the Radon measures implies that $|\mu|\left(V_{x_{\xi} \mid m} \backslash R_{\xi}\right)$ 's converge to 0 . Let $m_{1}$ be such that

$$
|\mu|\left(V_{x_{\xi} \mid m} \backslash R_{\xi}\right) \leq \frac{\varepsilon}{4\|f\|}
$$

for $m \geq m_{1}$.

Note also that a simple function is a linear combination of characteristic functions of clopen sets, hence there are $\xi_{1}, \ldots, \xi_{k-1}<\xi<\omega_{1}$ and $m_{2}$ such that the preimages under $f$ of each of its finite rational values belong to the subalgebra of $\mathcal{A}_{\xi+1}$ generated by $V_{t}$ 's for $|t|<m_{2}$ and $A_{\xi_{1}, j}, \ldots, A_{\xi_{k-1}, j}, A_{\xi, j}$ for $1 \leq j \leq N$. Now let $n \geq m_{1}, m_{2}$ be such that for every $1 \leq i<k$ there is $1 \leq j \leq N$ such that $V_{x_{\xi} \mid m} \subseteq A_{\xi_{i}, j}$, which can be obtained by the property (5) (of Definition 2.1) of $A_{\xi}$ 's and $\eta=\xi_{i}$.

It follows that $f$ is constant on $A_{\xi, j} \cap V_{x_{\xi} \mid m}$ for every $1 \leq j \leq N$. Let $q_{1}^{\prime}, \ldots, q_{N}^{\prime} \in \mathbb{Q}$ be the corresponding values and note that $\left|q_{l}^{\prime}-q_{N}^{\prime}\right| \leq 2\|f\|$ for any $1 \leq l \leq N$. So, by conditions (2) and (3) (of Definition 2.1) of $A_{\xi, j}$ 's we have

$$
f=\left[f \mid\left(K \backslash V_{x_{\xi} \mid m}\right)+q_{N}^{\prime} \chi_{V_{x_{\xi} \mid m}}\right]+\sum_{1 \leq l<N}\left(q_{l}^{\prime}-q_{N}^{\prime}\right) \chi_{A_{\xi, l} \cap V_{x_{\xi} \mid m}} .
$$

Note that $f \mid\left(K \backslash V_{x_{\xi} \mid m}\right)$ belongs to $C_{\xi}$ by Lemma 2.5, and so

$$
f=h+\sum_{1 \leq l<N} q_{l} \chi_{A_{\xi, l} \cap V_{x_{\xi} \mid m}}, \quad \max _{1 \leq l<N}\left|q_{l}\right||\mu|\left(V_{x_{\xi} \mid m} \backslash R_{\xi}\right) \leq \frac{\varepsilon}{2}
$$

where $q_{l}=q_{l}^{\prime}-q_{N}^{\prime}$ and $h \in C_{\xi}$. Hence the inductive assumption for $\varepsilon / 2$ can be used, which completes the proof of the lemma.

Definition 2.7. We say that an $N$-splitting family $\left(A_{\xi, i}: \xi<\omega_{1}\right.$, $i \in[N])$ is balanced if it satisfies the following additional condition:

(6) for all distinct $\xi, \eta \in \omega_{1}$ and all $j \in[2 n]$,

$$
\begin{aligned}
\mid\{i \in\{1,3, \ldots, 2 n-1\}: & \left.\left(x_{\eta}, i\right) \in A_{\xi, j}\right\} \mid \\
& =\left|\left\{i \in\{2,4, \ldots, 2 n\}:\left(x_{\eta}, i\right) \in A_{\xi, j}\right\}\right| .
\end{aligned}
$$

Lemma 2.8. Suppose that $n \in \mathbb{N}$ and $K_{2 n}$ is an unordered $2 n$-split Cantor set, where the $N$-splitting family $\left(A_{\xi, i}: \xi<\omega_{1}, i \in[2 n]\right)$ is balanced. Then:

(a) $K_{2 n}^{n+1}$ contains an uncountable discrete subspace;

(b) there is an uncountable biorthogonal system in $C\left(K_{2 n}\right)$ with $2 n$-supported functionals.

Proof. To prove (a), let us show that the subset $\left\{\left(\left(x_{\xi}, 1\right),\left(x_{\xi}, 2\right)\right.\right.$, $\left.\left.\left(x_{\xi}, 4\right), \ldots,\left(x_{\xi}, 2 n\right)\right): \xi<\omega_{1}\right\}$ of $K_{2 n}^{n+1}$ is relatively discrete.

Let $U_{\xi}=A_{\xi, 1} \times A_{\xi, 2} \times A_{\xi, 4} \times \cdots \times A_{\xi, 2 n}$, which is clearly an open neighbourhood of $\left(\left(x_{\xi}, 1\right),\left(x_{\xi}, 2\right),\left(x_{\xi}, 4\right), \ldots,\left(x_{\xi}, 2 n\right)\right)$. Now, fix distinct $\xi, \eta<\omega_{1}$ and let us prove that $\left(\left(x_{\eta}, 1\right),\left(x_{\eta}, 2\right),\left(x_{\eta}, 4\right), \ldots,\left(x_{\eta}, 2 n\right)\right) \notin U_{\xi}$. 
For contradiction, suppose $\left(\left(x_{\eta}, 1\right),\left(x_{\eta}, 2\right),\left(x_{\eta}, 4\right), \ldots,\left(x_{\eta}, 2 n\right)\right) \in U_{\xi}$, that is, $\left(x_{\eta}, j\right) \in A_{\xi, j}$ for each $j=1,2,4, \ldots, 2 n$. By condition 2.7(6), we see that for each $j \in[2 n]$,

$$
\begin{aligned}
\mid\left\{i \in\{1,3, \ldots, 2 n-1\}:\left(x_{\eta}, i\right)\right. & \left.\in A_{\xi, j}\right\} \mid \\
& =\left|\left\{i \in\{2,4, \ldots, 2 n\}:\left(x_{\eta}, i\right) \in A_{\xi, j}\right\}\right| .
\end{aligned}
$$

Hence, each set $A_{\xi, 2}, A_{\xi, 4}, \ldots, A_{\xi, 2 n}$ must contain at least one of the $\left(x_{\eta}, 1\right)$, $\left(x_{\eta}, 3\right), \ldots,\left(x_{\eta}, 2 n-1\right)$. By the disjointness of the $A_{\xi, j}$ 's (property (2) of Definition 2.1),$\left(x_{\eta}, 1\right)$ has to be in one of the sets $A_{\xi, 2}, A_{\xi, 4}, \ldots, A_{\xi, 2 n}$. But by our assumption, $\left(x_{\eta}, 1\right) \in A_{\xi, 1}$ and again by the disjointness of the $A_{\xi, j}$ 's, this is a contradiction.

To show (b), for each $\xi<\omega_{1}$, let $f_{\xi}=\chi_{A_{\xi, 2 n}}$ and

$$
\mu_{\xi}=\sum_{k=1}^{n}\left(\delta_{\left(x_{\xi}, 2 i\right)}-\delta_{\left(x_{\xi}, 2 i-1\right)}\right)
$$

and note that $\left(f_{\xi}, \mu_{\xi}\right)_{\xi<\omega_{1}} \subseteq C\left(K_{2 n}\right) \times M\left(K_{2 n}\right)$. Let us prove that this is a biorthogonal system.

For each $\xi<\omega_{1}$, since $\left(x_{\xi}, i\right) \in A_{\xi, i}$ and these sets are disjoint (property (2) of Definition 2.1), we get

$$
\begin{aligned}
\mu_{\xi}\left(f_{\xi}\right) & =\sum_{k=1}^{n}\left(\delta_{\left(x_{\xi}, 2 k\right)}-\delta_{\left(x_{\xi}, 2 k-1\right)}\right)\left(\chi_{A_{\xi, 2 n}}\right) \\
& =\sum_{k=1}^{n}\left(\chi_{A_{\xi, 2 n}}\left(\left(x_{\xi}, 2 k\right)\right)-\chi_{A_{\xi, 2 n}}\left(\left(x_{\xi}, 2 k-1\right)\right)\right)=\chi_{A_{\xi, 2 n}}\left(\left(x_{\xi}, 2 n\right)\right)=1 .
\end{aligned}
$$

On the other hand, for distinct $\xi, \eta<\omega_{1}$, by property (6), we know that for all $j \in[2 n]$,

$\left|\left\{i \in\{1,3, \ldots, 2 n-1\}:\left(x_{\eta}, i\right) \in A_{\xi, j}\right\}\right|=\left|\left\{i \in\{2,4, \ldots, 2 n\}:\left(x_{\eta}, i\right) \in A_{\xi, j}\right\}\right|$.

Hence,

$$
\begin{aligned}
\mu_{\xi}\left(f_{\eta}\right)= & \sum_{k=1}^{n}\left(\delta_{\left(x_{\xi}, 2 k\right)}-\delta_{\left(x_{\xi}, 2 k-1\right)}\right)\left(\chi_{A_{\eta, 2 n}}\right) \\
= & \sum_{k=1}^{n}\left(\chi_{A_{\eta, 2 n}}\left(\left(x_{\xi}, 2 k\right)\right)-\chi_{A_{\eta, 2 n}}\left(\left(x_{\xi}, 2 k-1\right)\right)\right) \\
= & \sum_{k=1}^{n} \chi_{A_{\eta, 2 n}}\left(\left(x_{\xi}, 2 k\right)\right)-\sum_{k=1}^{n} \chi_{A_{\eta, 2 n}}\left(\left(x_{\xi}, 2 k-1\right)\right) \\
= & \left|\left\{i \in\{2,4, \ldots, 2 n\}:\left(x_{\xi}, i\right) \in A_{\eta, 2 n}\right\}\right| \\
& \quad-\left|\left\{i \in\{1,3, \ldots, 2 n-1\}:\left(x_{\xi}, i\right) \in A_{\eta, 2 n}\right\}\right|=0,
\end{aligned}
$$

showing that $\left(f_{\xi}, \mu_{\xi}\right)_{\xi<\omega_{1}} \subseteq C\left(K_{2 n}\right) \times M\left(K_{2 n}\right)$ is a biorthogonal system. 
3. The generic construction. This section is devoted to a generic construction of an unordered $2 n$-split Cantor set which exhibits quite random features. This type of uncountable structures was first investigated systematically in [19]. One can describe this random behaviour as: in any uncountable sequence of finite substructures there are two which are related as we wish (up to constraints). We fix an uncountable sequence $\left(x_{\xi}: \xi<\omega_{1}\right) \subseteq 2^{\omega}$ consisting of distinct elements.

Definition 3.1. Let $\mathbb{P}$ be the forcing formed by the conditions

$$
p=\left(F_{p}, n_{p},\left(f_{\xi}^{p}: \xi \in F_{p}\right)\right),
$$

where:

1. $F_{p} \in\left[\omega_{1}\right]^{<\omega}$;

2. $n_{p} \in \omega$ is such that for all $\xi \neq \eta$ in $F_{p}, x_{\xi}\left|n_{p} \neq x_{\eta}\right| n_{p}$;

3. for all $\xi \in F_{p}$,

$$
f_{\xi}^{p}: 2^{n_{p}} \backslash\left\{x_{\xi} \mid n_{p}\right\} \rightarrow[2 n]^{[2 n]} \times\left[F_{p} \cap(\xi+1)\right]
$$

is such that

(a) if $f_{\xi}^{p}(s)=(\varphi, \xi)$, then $\varphi$ is a constant function;

(b) if $f_{\xi}^{p}(s)=(\varphi, \eta)$ for some $\eta<\xi$, then

$$
\forall j \in[2 n] \quad\left|\varphi^{-1}(j) \cap\{1,3,5, \ldots, 2 n-1\}\right|=\left|\varphi^{-1}(j) \cap\{2,4, \ldots, 2 n\}\right| .
$$

We put $q \leq p$ if $F_{q} \supseteq F_{p}, n_{q} \geq n_{p}$ and for all $\xi \in F_{p}$, all $s \in 2^{n_{q}} \backslash\left\{x_{\xi} \mid n_{q}\right\}$ and all $t \in 2^{n_{p}} \backslash\left\{x_{\xi} \mid n_{p}\right\}$,

$$
t \subseteq s \Rightarrow f_{\xi}^{p}(t)=f_{\xi}^{q}(s) .
$$

Intuitively, we are of course trying to build a $2 n$-split Cantor set which is determined by the choice of the balanced $2 n$-splitting family formed by $A_{\xi, i}$ 's. Thus the coordinate $f_{\xi}^{p}(s)$ describes the behaviour of $A_{\xi, i}$ 's on $V_{s}$. The formal description is given in Definition 3.3. The value $f_{\xi}^{p}(s)=(\varphi, \xi)$, where $\varphi$ has to be a constant function, say equal to $i$, means that the entire $V_{s}$ is included in $A_{\xi, i}$. The value $f_{\xi}^{p}=(\varphi, \eta)$ for some $\eta<\xi$ means that $A_{\xi, i}$ 's divide $V_{s}$ as coded by $\varphi$, i.e. $A_{\eta, j} \cap V_{s} \subseteq A_{\xi, \varphi(j)}$ for each $j \in[N]$. Note that a condition $p \in \mathbb{P}$ carries no information about the behaviour of $A_{\xi, i}$ 's on $V_{x_{\xi} \mid n_{p}}$, other than $\left(x_{\xi}, i\right) \in A_{\xi, i}$. This is the degree of freedom we have and which can be controlled by passing to an appropriate extension $q \leq p$. Condition (b) is to guarantee that the family of $A_{\xi, i}$ 's is balanced, that is, satisfies property (6) of Definition 2.7.

Lemma 3.2. The following subsets of $\mathbb{P}$ are dense in $\mathbb{P}$ :

(i) $\left\{p \in \mathbb{P}: n_{p} \geq k\right\}$ for each fixed $k \in \mathbb{N}$;

(ii) $\left\{p \in \mathbb{P}: \xi \in F_{p}\right\}$ for each fixed $\xi<\omega_{1}$. 
Proof. For (i), fix $k \in \mathbb{N}$ and let $p=\left(F_{p}, n_{p},\left(f_{\xi}^{p}: \xi \in F_{p}\right)\right) \in \mathbb{P}$. If $n_{p}<k$, define $q=\left(F_{q}, n_{q},\left(f_{\xi}^{q}: \xi \in F_{q}\right)\right)$ by putting $F_{q}=F_{p}, n_{q}=k$ and for each $\xi \in F_{q}=F_{p}, f_{\xi}^{q}$ is any function satisfying condition 3 of the definition of the forcing such that $f_{\xi}^{q}(t)=f_{\xi}^{p}\left(t \mid n_{p}\right)$ if $t \mid n_{p} \in 2^{n_{p}} \backslash\left\{x_{\xi} \mid n_{p}\right\}$; for example, let

$$
f_{\xi}^{q}(t)= \begin{cases}f_{\xi}^{p}\left(t \mid n_{p}\right) & \text { if } t \mid n_{p} \in 2^{n_{p}} \backslash\left\{x_{\xi} \mid n_{p}\right\}, \\ (\varphi, \xi) & \text { otherwise, }\end{cases}
$$

where $\varphi$ is the constant function equal to 1 . It is easy to see that $q \in \mathbb{P}$ and $q \leq p$.

For (ii), fix $\xi<\omega_{1}$ and let $p=\left(F_{p}, n_{p},\left(f_{\xi}^{p}: \xi \in F_{p}\right)\right) \in \mathbb{P}$. By (i), we may assume that $n_{p}$ is such that $x_{\eta}\left|n_{p} \neq x_{\xi}\right| n_{p}$ for all $\eta \in F_{p}$. Define $q=\left(F_{q}, n_{q},\left(f_{\xi}^{q}: \xi \in F_{q}\right)\right)$ by putting $F_{q}=F_{p} \cup\{\xi\}, n_{q}=n_{p}, f_{\eta}^{q}=f_{\eta}^{p}$ for each $\eta \in F_{p}$, and $f_{\xi}^{q}$ is any function satisfying condition 3 of the definition of the forcing; for example, let $f_{\xi}^{q}(t)=(\varphi, \xi)$, where $\varphi$ is the constant function equal to 1 . It is easy to see that $q \in \mathbb{P}$ and $q \leq p$.

Definition 3.3. Given a $\mathbb{P}$-generic filter $G$ over a model $V$, we define the family $\left\{A_{\xi, j}: \xi \in \omega_{1}, j \in[2 n]\right\}$ as follows: for each $\xi \in \omega_{1}$ and each $j \in[2 n]$, let

$A_{\xi, j}=\bigcup\left\{V_{s} \cap A_{\eta, i}: \exists p \in G, f_{\xi}^{p}(s)=(\varphi, \eta)\right.$ for some $\eta \neq \xi$ and $\left.\varphi(i)=j\right\}$

$\cup \bigcup\left\{V_{s}: \exists p \in G, f_{\xi}^{p}(s)=(\varphi, \xi)\right.$ and $\varphi$ is the constant function equal to $\left.j\right\}$

$\cup\left\{\left(x_{\xi}, j\right)\right\}$.

The following lemma follows directly from the above definition.

Lemma 3.4. Given $p \in G, \xi \in F_{p}$ and $s \in 2^{n_{p}} \backslash\left\{x_{\xi} \mid n_{p}\right\}$, we have:

(a) if $f_{\xi}^{p}(s)=(\varphi, \xi)$, then $V_{s} \subseteq A_{\xi, j}$ for $j=\varphi(1)$;

(b) if $f_{\xi}^{p}(s)=(\varphi, \eta)$ for some $\eta<\xi$, then $\forall i \in[2 n], V_{s} \cap A_{\eta, i} \subseteq A_{\xi, \varphi(i)}$.

Notice that in case $f_{\xi}^{p}(s)=(\varphi, \xi), \varphi$ is the constant function equal to $j$, so that we could have taken $j=\varphi(i)$ for any $i \in[2 n]$.

Let us now check that the family $\left\{A_{\xi, j}: \xi \in \omega_{1}, j \in[2 n]\right\}$ has the desired properties.

Theorem 3.5. The family $\left\{A_{\xi, j}: \xi \in \omega_{1}, j \in[2 n]\right\}$ is a balanced $2 n$ splitting family.

Proof. Let us prove that the family satisfies conditions $2.1(1)-(5)$ and $2.7(6)$.

(1) follows directly from the definition of $A_{\xi, j}$.

(2) This is proved by induction on $\xi$. First notice that by the definition of the forcing $\mathbb{P}$,

$$
\forall p \in \mathbb{P} \forall \xi \in F_{p} \forall s \in \operatorname{dom} f_{\xi}^{p} \quad R_{\xi} \cap V_{s}=\emptyset,
$$

since $x_{\xi} \mid n_{p} \notin \operatorname{dom} f_{\xi}^{p}$. Thus, $\left(x_{\xi}, j_{1}\right) \in A_{\xi, j_{2}}$ iff $j_{1}=j_{2}$. 
Now, fix $\xi<\omega_{1}$ and suppose $A_{\eta, i}$ are pairwise disjoint for each fixed $\eta<\xi$. Suppose there is $x \in A_{\xi, j_{1}} \cap A_{\xi, j_{2}}$ for some distinct $j_{1}, j_{2} \in[2 n]$. By the above observation, $x \neq\left(x_{\xi}, j\right)$ for any $j \in[2 n]$.

By the definition of $A_{\xi, j_{k}}$, for each $k \in\{1,2\}$ there are $p_{k} \in G$ and $s_{k} \in \operatorname{dom} f_{\xi}^{p_{k}}$ such that $x \in V_{s_{k}}$ and either

- $f_{\xi}^{p_{k}}\left(s_{k}\right)=\left(\varphi_{k}, \xi\right)$ and $\varphi_{k}$ is the constant function equal to $j_{k}$, or

- $f_{\xi}^{p_{k}}\left(s_{k}\right)=\left(\varphi_{k}, \eta_{k}\right)$ for some $\eta_{k}<\xi$ and $x \in A_{\eta_{k}, i}$ for some $i \in \varphi_{k}^{-1}\left(j_{k}\right)$.

Let $p \in G$ be such that $p \leq p_{1}, p_{2}$ and let $t \in 2^{n_{p}} \backslash\left\{x_{\xi} \mid n_{p}\right\}$ be such that $x \in V_{t}$. Then $t \supseteq s_{k}$ since $x \in V_{s_{k}}$, and hence, by the definition of extension in $\mathbb{P}, f_{\xi}^{p_{1}}\left(s_{1}\right)=f_{\xi}^{p}(t)=f_{\xi}^{p_{2}}\left(s_{2}\right)$, so that $\varphi_{1}=\varphi_{2}$.

Now, if $f_{\xi}^{p}(t)=(\varphi, \xi)$, this would mean that $\varphi_{1}$ and $\varphi_{2}$ are both constant equal to $j_{1}$ and $j_{2}$, contradicting the hypothesis that $j_{1} \neq j_{2}$. Otherwise, if $f_{\xi}^{p}(t)=(\varphi, \eta)$, for some $\eta<\xi$, we would get $x \in A_{\eta, i_{k}}$ for some $i_{k} \in \varphi^{-1}\left(j_{k}\right)$. By the inductive hypothesis $i_{1}=i_{2} \in \varphi^{-1}\left(j_{1}\right) \cap \varphi^{-1}\left(j_{2}\right)$, which implies that $j_{1}=j_{2}$, again contradicting the hypothesis.

This concludes the proof that the family satisfies condition (2) of Definition 2.1.

(3) is again proved by induction on $\xi$. So, let $\xi<\omega_{1}$, suppose $K=$ $A_{\eta, 1} \cup \cdots \cup A_{\eta, 2 n}$ for any $\eta<\xi$ and let $x \in K$.

If $x=\left(x_{\xi}, i\right)$ for some $i \in[2 n]$, then $x \in A_{\xi, i}$ by definition.

By Lemma 3.2, let $p \in G$ be such that $x \in V_{s}$ for some $s \in 2^{n_{p}} \backslash\left\{x_{\xi} \mid n_{p}\right\}$.

If $f_{\xi}^{p}(s)=(\varphi, \xi)$, by Lemma 3.4(a) we get $V_{s} \subseteq A_{\xi, \varphi(1)}$, which guarantees that $x \in A_{\xi, \varphi(1)}$.

Otherwise, if $f_{\xi}^{p}(s)=(\varphi, \eta)$ for some $\eta<\xi$, by the inductive hypothesis, let $i \in[2 n]$ be such that $x \in A_{\eta, i}$. Then, by Lemma 3.4 (b), $V_{s} \cap A_{\eta, i} \subseteq A_{\xi, \varphi(i)}$, which implies that $x \in A_{\xi, \varphi(i)}$ and concludes the proof of condition (3) of Definition 2.1.

To prove (4), fix $\eta<\xi<\omega_{1}$ and $i \in[2 n]$. By Lemma 3.2, let $p \in G$ be such that $\xi, \eta \in F_{p}$ and $x_{\eta}\left|n_{p} \neq x_{\xi}\right| n_{p}$.

If $f_{\xi}^{p}\left(x_{\eta} \mid n_{p}\right)=(\varphi, \xi)$, by Lemma 3.4(a) we get $V_{x_{\eta} \mid n_{p}} \subseteq A_{\xi, \varphi(1)}$ (and in particular $\left.V_{x_{\eta} \mid n_{p}} \cap A_{\eta, i} \subseteq V_{x_{\eta} \mid n_{p}} \cap A_{\xi, \varphi(1)}\right)$.

If $f_{\xi}^{p}\left(x_{\eta} \mid n_{p}\right)=(\varphi, \eta)$ for some $\eta<\xi$, then, by Lemma 3.4 (b), we have $V_{x_{\eta} \mid n_{p}} \cap A_{\eta, i} \subseteq A_{\xi, \varphi(i)}$ (and in particular $V_{x_{\eta} \mid n_{p}} \cap A_{\eta, i} \subseteq V_{x_{\eta} \mid n_{p}} \cap A_{\xi, \varphi(i)}$ ), and we are done by condition (4) of Definition 2.1 .

(5) is proved by induction on $\xi<\omega_{1}$. Let $\xi<\omega_{1}$ and $x \in 2^{\omega} \backslash\left\{x_{\eta}: \eta \leq \xi\right\}$.

If $x=x_{\eta}$ for some $\eta>\xi$, by Lemma 3.2 there is $p \in G$ such that $\xi, \eta \in F_{p}$. Otherwise, if $x \in 2^{\omega} \backslash\left\{x_{\eta}: \eta<\omega_{1}\right\}$, by Lemma 3.2 there is $p \in G$ such that $\xi \in F_{p}$ and $x\left|n_{p} \neq x_{\xi}\right| n_{p}$. In both cases, put $s=x \mid n_{p}$.

If $f_{\xi}^{p}(s)=(\varphi, \xi)$, then, by Lemma 3.4 (a), $V_{s} \subseteq A_{\xi, \varphi(1)}$. 
If $f_{\xi}^{p}(s)=\left(\varphi, \eta^{\prime}\right)$ for some $\eta^{\prime} \in F_{p} \cap \xi$, by the inductive hypothesis, there are $k \in \mathbb{N}$ and $i \in[2 n]$ such that $V_{x \mid k} \subseteq A_{\eta^{\prime}, i}$. By Lemma 3.2, let $q \in G$ be such that $q \leq p$ and $n_{q} \geq k$. Putting $t=x \mid n_{q}$, we get $V_{t} \subseteq V_{x \mid k} \subseteq A_{\eta^{\prime}, i}$ and $f_{\xi}^{q}(t)=f_{\xi}^{p}(s)=\left(\varphi, \eta^{\prime}\right)$, since $t \supseteq s$. This implies by Lemma 3.4(b) that $V_{t}=V_{t} \cap A_{\eta^{\prime}, i} \subseteq A_{\xi, \varphi(i)}$, which concludes the proof of condition (5) of Definition 2.1.

Hence, the family formed by the $A_{\xi, i}$ 's is a $2 n$-splitting family.

(6) is proved by induction on $\xi<\omega_{1}$. So, fix $\xi<\omega_{1}$ and suppose we know that for all $\zeta<\xi$, all $\eta \neq \zeta$ and all $j \in[2 n]$, $\left|\left\{i \in\{1,3, \ldots, 2 n-1\}:\left(x_{\eta}, i\right) \in A_{\zeta, j}\right\}\right|=\left|\left\{i \in\{2,4, \ldots, 2 n\}:\left(x_{\eta}, i\right) \in A_{\zeta, j}\right\}\right|$.

Now, fix $\eta \neq \xi$. Let $p \in G$ be such that $\xi, \eta \in F_{p}$, so that $x_{\eta} \mid n_{p} \in \operatorname{dom} f_{\xi}^{p}$.

If $f_{\xi}^{p}\left(x_{\eta} \mid n_{p}\right)=(\varphi, \xi)$, then, by Lemma $3.4(\mathrm{a}), V_{x_{\eta} \mid n_{p}} \subseteq A_{\xi, \varphi(1)}$, which implies that $\left(x_{\eta}, i\right) \in A_{\xi, \varphi(1)}$ for all $i \in[2 n]$. By the disjointness of the $A_{\xi, i}$ 's, (3) and condition (6) of Definition 2.7 hold both for $A_{\xi, \varphi(1)}$ (which contains all $\left.\left(x_{\eta}, i\right)\right)$ and for $A_{\xi, j}, j \neq \varphi(1)$ (which contain no $\left(x_{\eta}, i\right)$ ).

If $f_{\xi}^{p}\left(x_{\eta} \mid n_{p}\right)=(\varphi, \zeta)$ for some $\zeta<\xi$ in $F_{p}$, then for all $i \in[2 n]$, we have $V_{x_{\eta} \mid n_{p}} \cap A_{\zeta, i} \subseteq A_{\xi, \varphi(i)}$. This means that each $A_{\xi, j}$ contains exactly those $\left(x_{\eta}, k\right)$ which are in $A_{\zeta, i}$ for some $i \in \varphi^{-1}(j)$. In particular,

$$
\begin{aligned}
& \left\{k \in\{1,3, \ldots, 2 n-1\}:\left(x_{\eta}, k\right) \in A_{\xi, j}\right\} \\
& \quad=\left\{k \in\{1,3, \ldots, 2 n-1\}:\left(x_{\eta}, k\right) \in A_{\zeta, i} \text { for some } i \in \varphi^{-1}(j)\right\} \\
& \quad=\bigcup_{i \in \varphi^{-1}(j)}\left\{k \in\{1,3, \ldots, 2 n-1\}:\left(x_{\eta}, k\right) \in A_{\zeta, i}\right\}
\end{aligned}
$$

and

$$
\begin{aligned}
\left\{k \in\{2,4, \ldots, 2 n\}:\left(x_{\eta}, k\right) \in A_{\xi, j}\right\} & \\
& =\left\{k \in\{2,4, \ldots, 2 n\}:\left(x_{\eta}, k\right) \in A_{\zeta, i} \text { for some } i \in \varphi^{-1}(j)\right\} \\
& =\bigcup_{i \in \varphi^{-1}(j)}\left\{k \in\{2,4, \ldots, 2 n\}:\left(x_{\eta}, k\right) \in A_{\zeta, i}\right\} .
\end{aligned}
$$

Let us now consider two cases:

If $\eta=\zeta$, since $\left(x_{\eta}, k\right) \in A_{\eta, k}$, we get

$\left\{k \in\{1,3, \ldots, 2 n-1\}:\left(x_{\eta}, k\right) \in A_{\xi, j}\right\}=\left\{k \in\{1,3, \ldots, 2 n-1\}: k \in \varphi^{-1}(j)\right\}$ and

$$
\left\{k \in\{2,4, \ldots, 2 n\}:\left(x_{\eta}, k\right) \in A_{\xi, j}\right\}=\left\{k \in\{2,4, \ldots, 2 n\}: k \in \varphi^{-1}(j)\right\} .
$$

By property $3(\mathrm{~b})$ of the definition of the partial ordering, the sets on the right-hand side of these two equalities have the same size, which guarantees $\left|\left\{k \in\{1,3, \ldots, 2 n-1\}:\left(x_{\eta}, k\right) \in A_{\xi, j}\right\}\right|=\left|\left\{k \in\{2,4, \ldots, 2 n\}:\left(x_{\eta}, k\right) \in A_{\xi, j}\right\}\right|$, concluding the proof in this case. 
If $\eta \neq \zeta$, by the inductive hypothesis we know that for all $i \in[2 n]$, $\left|\left\{k \in\{1,3, \ldots, 2 n-1\}:\left(x_{\eta}, k\right) \in A_{\zeta, i}\right\}\right|=\left|\left\{k \in\{2,4, \ldots, 2 n\}:\left(x_{\eta}, k\right) \in A_{\zeta, i}\right\}\right|$. Hence,

$$
\begin{aligned}
\mid\{k \in\{1,3, \ldots, 2 n & \left.-1\}:\left(x_{\eta}, k\right) \in A_{\xi, j}\right\} \mid \\
& =\left|\bigcup_{i \in \varphi^{-1}(j)}\left\{k \in\{1,3, \ldots, 2 n-1\}:\left(x_{\eta}, k\right) \in A_{\zeta, i}\right\}\right| \\
& =\left|\bigcup_{i \in \varphi^{-1}(j)}\left\{k \in\{2,4, \ldots, 2 n\}:\left(x_{\eta}, k\right) \in A_{\zeta, i}\right\}\right| \\
& =\left|\left\{k \in\{2,4, \ldots, 2 n\}:\left(x_{\eta}, k\right) \in A_{\xi, j}\right\}\right|,
\end{aligned}
$$

which concludes the proof of condition (6) of Definition 2.7, that is, the family of $A_{\xi, i}$ 's is a balanced $2 n$-splitting family.

Proposition 3.6. Let $p_{1}=\left(F_{1}, n_{1},\left(f_{\xi}^{1}: \xi \in F_{1}\right)\right)$ and $p_{2}=\left(F_{2}, n_{2}\right.$, $\left.\left(f_{\xi}^{2}: \xi \in F_{2}\right)\right)$ be conditions of $\mathbb{P}$ such that:

- $F_{1} \cap F_{2}<F_{1} \backslash F_{2}<F_{2} \backslash F_{1}$;

- $n_{1}=n_{2}=n$;

- there is an order-preserving bijection $e: F_{1} \rightarrow F_{2}$ such that

- for all $\xi \in F_{1}, x_{\xi}\left|n=x_{e(\xi)}\right| n$;

- for all $\xi \in F_{1}$ and all $s \in 2^{n_{1}} \backslash\left\{x_{\xi} \mid n_{1}\right\}\left(=2^{n_{2}} \backslash\left\{x_{e(\xi)} \mid n_{2}\right\}\right)$,

$$
f_{e(\xi)}^{2}(s)=(\varphi, e(\eta)) \text { where } f_{\xi}^{1}(s)=(\varphi, \eta) \text {. }
$$

Then, given $\left(\epsilon_{\xi}: \xi \in F_{1} \backslash F_{2}\right) \subseteq[2 n]^{[2 n]}$ such that for all $\xi \in F_{1} \backslash F_{2}$,

$$
\forall j \in[2 n] \quad\left|\epsilon_{\xi}^{-1}(j) \cap\{1,3,5, \ldots, 2 n-1\}\right|=\left|\epsilon_{\xi}^{-1}(j) \cap\{2,4,6, \ldots, 2 n\}\right|,
$$

and given constant functions $\left(\delta_{\xi}: \xi \in F_{1} \backslash F_{2}\right) \subseteq[2 n]^{[2 n]}$, there is $q \leq p_{1}, p_{2}$, $q \in \mathbb{P}$, such that

$$
\forall \xi \in F_{1} \backslash F_{2} \quad f_{\xi}^{q}\left(x_{e(\xi)} \mid n_{q}\right)=\left(\delta_{\xi}, \xi\right) \quad \text { and } \quad f_{e(\xi)}^{q}\left(x_{\xi} \mid n_{q}\right)=\left(\epsilon_{\xi}, \xi\right) .
$$

Proof. Define $q=\left(F_{q}, n_{q},\left(f_{\xi}^{q}: \xi \in F_{q}\right)\right)$ as follows: let $F_{q}=F_{1} \cup F_{2}$; let $n_{q} \in \mathbb{N}$ be such $n_{p} \leq n_{q}$ and for all $\xi<\eta \in F_{q}, x_{\xi}\left|n_{q} \neq x_{\eta}\right| n_{q}$; for each $\xi \in F_{q}$ and $t \in 2^{n_{q}} \backslash\left\{x_{\xi} \mid n_{q}\right\}$, let

$$
f_{\xi}^{q}(t)=\left\{\begin{array}{lll}
f_{\xi}^{1}(t \mid n) & \text { if } \xi \in F_{1} \text { and } t\left|n \neq x_{\xi}\right| n & \text { (Case 1), } \\
f_{\xi}^{2}(t \mid n) & \text { if } \xi \in F_{2} \text { and } t\left|n \neq x_{\xi}\right| n & \text { (Case 2), } \\
\left(\delta_{\xi}, \xi\right) & \text { if } \xi \in F_{1} \text { and } t\left|n=x_{\xi}\right| n & \text { (Case 3), } \\
\left(\epsilon_{e^{-1}(\xi)}, e^{-1}(\xi)\right) & \text { if } \xi \in F_{2} \backslash F_{1} \text { and } t\left|n=x_{\xi}\right| n & \text { (Case 4). }
\end{array}\right.
$$

$f_{\xi}^{q}$ is well-defined since $e(\xi)=\xi$ whenever $\xi \in F_{1} \cap F_{2}$, so that $f_{\xi}^{1}(s)=$ $f_{e(\xi)}^{2}(s)=f_{\xi}^{2}(s)$ for $s \in 2^{n} \backslash\left\{x_{\xi} \mid n\right\}$. 
Let us now prove that $q \in \mathbb{P}$. Conditions 1 and 2 of Definition 3.1 follow directly from the definition of $F_{q}$ and $n_{q}$.

To prove that $q$ satisfies condition 3 , fix $\xi \in F_{q}$ and $t \in 2^{n_{q}} \backslash\left\{x_{\xi} \mid n_{q}\right\}$. In Case 1 (resp. Case 2), both conditions 3(a) and 3(b) follow from the fact that $p_{1}$ (resp. $\left.p_{2}\right)$ is in $\mathbb{P}$.

In Case 3, we only have to check condition 3(a), which is guaranteed by the fact that $\left(\delta_{\xi}: \xi \in F_{1} \backslash F_{2}\right) \subseteq[2 n]^{[2 n]}$ are assumed to be constant.

Similarly, in Case 4, we only have to check condition 3(b), which is guaranteed by the fact that $\left(\epsilon_{\xi}: \xi \in F_{1} \backslash F_{2}\right) \subseteq[2 n]^{[2 n]}$ are assumed to be as needed.

Let us now prove that $q \leq p_{1}, p_{2}$. Trivially, $F_{1}, F_{2} \subseteq F_{q}$ and $n_{1}, n_{2} \leq n_{q}$.

Given $\xi \in F_{q}, s \in 2^{n} \backslash\left\{x_{\xi} \mid n\right\}$ and $t \in 2^{n_{q}} \backslash\left\{x_{\xi} \mid n_{q}\right\}$ such that $s \subseteq t$, let $k \in\{1,2\}$ be such that $\xi \in F_{k}$ and notice that we are in Cases 1 or 2 , since $t \mid n=s$. Therefore, $f_{\xi}^{q}(t)=f_{\xi}^{k}(t \mid n)=f_{\xi}^{k}(s)$, which implies that $q \leq p_{1}, p_{2}$.

Finally, notice that the definition of $f_{\xi}^{q}(t)$ in Cases 1 or 2 implies $(3.1)$.

Theorem 3.7. $\mathbb{P}$ is c.c.c.

Proof. For each $\alpha<\omega_{1}$, let $p_{\alpha}=\left(F_{\alpha}, n_{\alpha},\left(f_{\eta}^{\alpha}\right)_{\eta \in F_{\alpha}}\right) \in \mathbb{P}$.

By the $\Delta$-system lemma, we can assume that $\left(F_{\alpha}\right)_{\alpha<\omega_{1}}$ forms a $\Delta$-system with root $\Delta$ such that for every $\alpha<\beta<\omega_{1}$,

- $\Delta<F_{\alpha} \backslash \Delta<F_{\beta} \backslash \Delta$ and $\left|F_{\alpha}\right|=\left|F_{\beta}\right|$.

Since each $n_{\alpha} \in \mathbb{N}$, we can suppose that for every $\alpha<\beta<\omega_{1}$,

- $n_{\alpha}=n_{\beta}=n$.

Also, we may assume that if $e_{\alpha \beta}: F_{\alpha} \rightarrow F_{\beta}$ is the order-preserving bijective function, then

- for all $\xi \in F_{\alpha}, x_{\xi}\left|n=x_{e_{\alpha \beta}(\xi)}\right| n$ (since both belong to $2^{n}$ );

- for all $\xi \in F_{\alpha}$ and all $s \in 2^{n} \backslash\left\{x_{\xi} \mid n\right\}$,

$$
f_{e_{\alpha \beta}(\xi)}^{\beta}(s)=\left(\varphi, e_{\alpha \beta}(\eta)\right), \quad \text { where } f_{\xi}^{\alpha}(s)=(\varphi, \eta) .
$$

Now, fix $\alpha<\beta<\omega_{1}$. Note that $p_{\alpha}$ and $p_{\beta}$ satisfy the hypothesis of Proposition 3.6. Let, for $\xi \in F_{\beta} \backslash \Delta, \epsilon_{\xi}$ be any function satisfying condition 3 of Definition 3.1 (for example, $\epsilon_{\xi}$ constant equal to 1); and for $\xi \in F_{\alpha} \backslash \Delta$, let $\delta_{\xi} \in[2 n]^{[2 n]}$ be any constant function. Then, by Proposition 3.6, there is $q \leq p_{\alpha}, p_{\beta}$ in $\mathbb{P}$, which concludes the proof.

THEOREM 3.8. Let $n \geq 1$ be a natural number. It is consistent that there is a compact Hausdorff totally disconnected space $K$ which is an unordered $2 n$-split Cantor set corresponding to a balanced $2 n$-splitting family $\left(A_{\xi, i}\right.$ : $\left.\xi<\omega_{1}, i \in[2 n]\right)$ such that given any collection of pairwise disjoint sets $E_{\alpha}=\left\{\xi_{\alpha}^{1}, \ldots, \xi_{\alpha}^{k}\right\} \subseteq \omega_{1}$ for $\alpha<\omega_{1}$, given $\epsilon:[k] \times[2 n] \rightarrow[2 n]$ such that $|\{l \in\{1,3,5, \ldots, 2 n-1\}: \epsilon(i, l)=j\}|=|\{l \in\{2,4,6, \ldots, 2 n\}: \epsilon(i, l)=j\}|$, 
and given $\delta:[k] \rightarrow[n]$, there are $\alpha<\beta$ such that for all $1 \leq i \leq k$,

$$
R_{\xi_{\beta}^{i}} \subseteq A_{\xi_{\alpha}^{i}, \delta(i)} \quad \text { and } \quad\left(x_{\xi_{\alpha}^{i}}, l\right) \in A_{\xi_{\beta}^{i}, \epsilon(i, l)} .
$$

Proof. By Theorem 3.5, $\mathbb{P}$ forces that $\left(A_{\xi, i}: \xi<\omega_{1}, i \in[2 n]\right)$ as in Definition 3.3 is a balanced $2 n$-splitting family. By Proposition 2.3 , the corresponding unordered $2 n$-split Cantor set is a compact, Hausdorff, totally disconnected space. Let us now prove the remaining desired property.

In $V$, suppose $\left(\dot{E}_{\alpha}\right)_{\alpha<\omega_{1}}$ and $\left(\dot{\xi}_{\alpha}^{i}\right)_{\alpha<\omega_{1}, 1 \leq i \leq k}$ are sequences of names such that $\mathbb{P}$ forces that $\dot{E}_{\alpha}=\left\{\dot{\xi}_{\alpha}^{1}<\cdots<\dot{\xi}_{\alpha}^{k}\right\}$ and $\left(\dot{E}_{\alpha}\right)_{\alpha<\omega_{1}}$ is pairwise disjoint.

For each $\alpha<\omega_{1}$, let $p_{\alpha}=\left(F_{\alpha}, n_{\alpha},\left(f_{\eta}^{\alpha}\right)_{\eta \in F_{\alpha}}\right) \in \mathbb{P}, \xi_{\alpha}^{1}, \ldots, \xi_{\alpha}^{k} \in \omega_{1}$ and $E_{\alpha}, \ldots, E_{\alpha} \subseteq \omega_{1}$ be finite such that

$$
p_{\alpha} \Vdash \forall 1 \leq i \leq k \quad \dot{\xi}_{\alpha}^{i}=\check{\xi}_{\alpha}^{i} \text { and } \dot{E}_{\alpha}=\check{E}_{\alpha} .
$$

By Lemma 3.2, we can assume without loss of generality that for all $\alpha<\omega_{1}, E_{i}^{\alpha} \subseteq F_{\alpha}$.

By the $\Delta$-system lemma, we can assume as well that $\left(F_{\alpha}\right)_{\alpha<\omega_{1}}$ forms a $\Delta$-system with root $\Delta$ such that for every $\alpha<\beta<\omega_{1}$,

- $\Delta<F_{\alpha} \backslash \Delta<F_{\beta} \backslash \Delta$ and $\left|F_{\alpha}\right|=\left|F_{\beta}\right|$.

Since each $n_{\alpha} \in \mathbb{N}$, we can suppose that for every $\alpha<\beta<\omega_{1}$,

- $n_{\alpha}=n_{\beta}=n$.

Also, we may assume that if $e_{\alpha \beta}: F_{\alpha} \rightarrow F_{\beta}$ is the order-preserving bijective function, then

- for all $\xi \in F_{\alpha}, x_{\xi}\left|n=x_{e_{\alpha \beta}(\xi)}\right| n$ (since both belong to $2^{n}$ );

- for all $\xi \in F_{\alpha}$ and all $s \in 2^{n} \backslash\left\{x_{\xi} \mid n\right\}$,

$$
f_{e_{\alpha \beta}(\xi)}^{\beta}(s)=\left(\varphi, e_{\alpha \beta}(\eta)\right), \quad \text { where } \quad f_{\xi}^{\alpha}(s)=(\varphi, \eta) .
$$

- for all $1 \leq i \leq k, e_{\alpha \beta}\left(\xi_{\alpha}^{i}\right)=\xi_{\beta}^{i}$.

Finally, we may assume that for all $1 \leq i \leq k$ we have: either $\xi_{\alpha}^{i}=\xi_{\beta}^{i}$ for all $\alpha<\beta<\omega_{1}$; or $\xi_{\alpha}^{i} \notin \Delta$ for all $\alpha<\omega_{1}$, and actually the second case holds by the assumption that $E_{\alpha}$ 's are pairwise disjoint.

Now, fix $\alpha<\beta<\omega_{1}$. Note that $p_{\alpha}$ and $p_{\beta}$ satisfy the hypothesis of Proposition 3.6. Taking $\epsilon_{\xi_{\beta}^{i}}=\epsilon(i, \cdot)$ and $\delta_{\xi_{\alpha}^{i}}=\delta(i)$ (and for $\xi \in F_{\beta} \backslash\left(\Delta \cup E_{\beta}\right)$, any function $\epsilon_{\xi}$ satisfying condition 3 of Definition 3.1, while for $\xi \in F_{\alpha} \backslash$ $\left(\Delta \cup E_{\alpha}\right)$, any constant function $\left.\delta_{\xi} \in[2 n]^{[2 n]}\right)$, by Proposition 3.6. there is $q \leq p_{\alpha}, p_{\beta}$ in $\mathbb{P}$ such that

$$
\forall \xi \in F_{\alpha} \backslash \Delta \quad f_{\xi}^{q}\left(x_{e_{\alpha \beta}(\xi)} \mid n_{q}\right)=\left(\delta_{\xi}, \xi\right) \quad \text { and } \quad f_{e_{\alpha \beta}(\xi)}^{q}\left(x_{\xi} \mid n_{q}\right)=\left(\epsilon_{e_{\alpha \beta}(\xi)}, \xi\right) \text {. }
$$

In particular, for all $1 \leq i \leq k$,

$$
f_{\xi_{\alpha}^{i}}^{q}\left(x_{\xi_{\beta}^{i}} \mid n_{q}\right)=\left(\delta(i), \xi_{\alpha}^{i}\right) \quad \text { and } \quad f_{\xi_{\beta}^{i}}^{q}\left(x_{\xi_{\alpha}^{i}} \mid n_{q}\right)=\left(\epsilon(i, \cdot), \xi_{\alpha}^{i}\right) .
$$


By the definition of $A_{\xi, j}$, we see that for all $1 \leq i \leq k$,

$$
R_{\xi_{\beta}^{i}} \subseteq A_{\xi_{\alpha}^{i}, \delta(i)} \quad \text { and } \quad\left(x_{\xi_{\alpha}^{i}}, l\right) \in A_{\xi_{\beta}^{i}, \epsilon(i, l)},
$$

which concludes the proof.

The fact that $2 n$ is even is exploited in the above proof. It turns out that there cannot be an analogue of an unordered $N$-split Cantor set for $N=3$ which behaves as in Theorem 3.8, since we have the following:

Lemma 3.9. Let $N \geq 3$ be a natural number. Suppose that $K$ is an unordered $N$-split Cantor set corresponding to an $N$-splitting family $\left(A_{\xi, i}: \xi<\omega_{1}, i \in[N]\right)$ such that given any sequence $\left(\xi_{\alpha}: \alpha<\omega_{1}\right)$ of distinct ordinals and $j \in[N]$, there are $\alpha<\beta$ such that

$$
R_{\xi_{\beta}} \subseteq A_{\xi_{\alpha}, j} .
$$

Suppose that $\left(f_{\alpha}, \mu_{\alpha}\right)_{\alpha<\omega_{1}}$ is a biorthogonal system such that $f_{\alpha}=\chi_{A_{\alpha}}$ for some clopen subset $A_{\alpha} \subseteq K$ and $\mu_{\alpha}=r_{\alpha} \delta_{\left(x_{\eta_{\alpha}}, 1\right)}+s_{\alpha} \delta_{\left(x_{\eta_{\alpha}}, 2\right)}+t_{\alpha} \delta_{\left(x_{\eta_{\alpha}}, 3\right)}$ for all $\alpha<\omega_{1}$, for some reals $r_{\alpha}, s_{\alpha}, t_{\alpha}$ and some sequence $\left(\eta_{\alpha}: \alpha<\omega_{1}\right)$. Then there is an uncountable nice biorthogonal system in $C(K)$.

Proof. If there is a biorthogonal system of the form $\left(\chi_{A_{\alpha}}, r_{\alpha} \delta_{y_{\alpha}}\right)$ for $\alpha<$ $\omega_{1}$ and $y_{\alpha} \in K$, then $r_{\alpha}=1$ for all $\alpha<\omega_{1}$ and $y_{\alpha} \notin A_{\beta}$ for any $\beta \neq \alpha$ and $y_{\alpha} \in A_{\alpha}$. So $\left(\chi_{A_{\alpha+1}}, \delta_{y_{\alpha+1}}-\delta_{y_{\alpha}}\right)$, say, for all limit ordinals $\alpha$ is a nice biorthogonal system.

If there is a biorthogonal system of the form $\left(\chi_{A_{\alpha}}, r_{\alpha} \delta_{y_{\alpha}}+s_{\alpha} \delta_{z_{\alpha}}\right)$ for $\alpha<\omega_{1}$ and $y_{\alpha}, z_{\alpha} \in K$, and $r_{\alpha}, s_{\alpha}, r_{\alpha}+s_{\alpha} \neq 0$, then $r_{\alpha}, s_{\alpha} \notin A_{\beta}$ for any $\alpha \neq \beta$ and a similar argument to the one above gives a nice biorthogonal system. If $r_{\alpha}+s_{\alpha}=0$ and $r_{\alpha}, s_{\alpha} \neq 0$, we may assume that $r_{\alpha}>0$ and so $s_{\alpha}=-r_{\alpha}$. It follows from the fact that $\left(r_{\alpha} \delta_{y_{\alpha}}+s_{\alpha} \delta_{z_{\alpha}}\right)\left(\chi_{A_{\alpha}}\right)=1$ that $r_{\alpha}=1$ and $s_{\alpha}=-1$, and so we have a nice biorthogonal system.

Hence, without loss of generality, we may assume that $r_{\alpha}, s_{\alpha}, t_{\alpha} \neq 0$ for all $\alpha<\omega_{1}$. First let us see that there is an uncountable $X \subseteq \omega_{1}$ such that $r_{\alpha}+s_{\alpha}+t_{\alpha}=0$ for all $\alpha \in X$. If not, then there is an uncountable $X \subseteq \omega_{1}$ and an $\varepsilon>0$ such that $\left|r_{\alpha}+s_{\alpha}+t_{\alpha}\right|>\varepsilon$ for each $\alpha \in X$.

Now note that as $\mu_{\alpha}\left(\chi_{A_{\alpha}}\right)=1 \neq 0$, we have $j \in\{1,2,3\}$ such that $\left(x_{\eta_{\alpha}}, j\right) \in A_{\alpha}$. We may assume that it is the same $j$ for all $\alpha \in X$. By the form of the basic neighbourhoods of points $\left(x_{\eta_{\alpha}}, j\right)$ we have $s \in 2^{m}$ for some $m \in \mathbb{N}$ such that $\left(x_{\eta_{\alpha}}, j\right) \in V_{s} \cap A_{\eta_{\alpha}, j} \subseteq A_{\alpha}$. We may assume that it is the same $s$ for all $\alpha \in X$. It follows that for some $n \in \mathbb{N}$ we have $s=x_{\eta_{\alpha}} \mid n$ for all $\alpha \in X$ and so $R_{\eta_{\alpha}} \subseteq V_{s}$ for all $\alpha \in X$. Apply the hypothesis of the lemma and obtain $\alpha<\beta$, both in $X$, such that $R_{\eta_{\beta}} \subseteq A_{\eta_{\alpha}, j}$; thus we get $R_{\eta_{\beta}} \subseteq V_{s} \cap A_{\eta_{\alpha}, j} \subseteq A_{\alpha}$. This means that $0=\mu_{\beta}\left(\chi_{A_{\alpha}}\right)=r_{\beta}+s_{\beta}+t_{\beta}$, contradicting the choice of $\beta \in X$. So we may assume that $r_{\alpha}+s_{\alpha}+t_{\alpha}=0$ for all $\alpha<\omega_{1}$. 
For three nonzero numbers whose sum is zero, there cannot be any subsum which is zero. This means that, for $\alpha \neq \beta$, as $\mu_{\alpha}\left(A_{\beta}\right)=0$, we have either $\left\{x_{\alpha}, y_{\alpha}, z_{\alpha}\right\} \cap A_{\beta}=\emptyset$ or $\left\{x_{\alpha}, y_{\alpha}, z_{\alpha}\right\} \subseteq A_{\beta}$. So, to make an uncountable nice biorthogonal system out of points $\left\{x_{\alpha}, y_{\alpha}, z_{\alpha}\right\}$ and functions $\chi_{A_{\alpha}}$, we need to find any fixed pair of them which is separated by $A_{\alpha}$ for uncountably many $\alpha$ 's.

But $A_{\alpha}$ must separate some pair as $\mu_{\alpha}\left(A_{\alpha}\right)=1$, so choose an uncountable subset $Y$ of $\omega_{1}$ on which the same pair is separated, say $x_{\alpha} \in A_{\alpha}$ and $z_{\alpha} \notin A_{\alpha}$.

Define $\nu_{\alpha}=\delta_{x_{\alpha}}-\delta_{z_{\alpha}}$ and note that $\left(\chi_{A_{\alpha}}, \nu_{\alpha}\right)_{\alpha \in Y}$ is an uncountable nice biorthogonal system.

\section{Biorthogonal and semibiorthogonal systems in $C\left(K_{2 n}\right)$ 's}

Lemma 4.1. Suppose that $\theta>\rho>0, n \in \mathbb{N}, n \geq 2$, and $r_{1}, \ldots, r_{2 n}$ are reals such that

(1) $\left|\sum_{1 \leq i \leq 2 n} r_{i}\right|<\rho$,

(2) there is $1 \leq i_{0} \leq 2 n$ such that $r_{i_{0}}>\theta$,

(3) there is $1 \leq i_{1} \leq 2 n$ such that $r_{i_{1}}=0$.

Then there are $1 \leq i, j \leq 2 n$ such that $(-1)^{i+j}=-1$ and

$$
r_{i}+r_{j}<\frac{2 n \rho-\theta}{n(2 n-2)} .
$$

Proof. By (1) and (2), since $\theta>\rho$, there must be an $i_{2} \in\{1, \ldots, 2 n\} \backslash$ $\left\{i_{0}, i_{1}\right\}$ such that

$$
r_{i_{2}}<-\frac{\theta-\rho}{2 n-2}=\frac{\rho-\theta}{2 n-2}<\frac{2 n \rho-\theta}{n(2 n-2)} .
$$

So, if there is $i_{3}$ such that $(-1)^{i_{2}+i_{3}}=-1$ and $r_{i_{3}} \leq 0$, then we are done. Otherwise, there are at least $n$ positive numbers $r_{i}$ (at least for all $i$ of parity other than $i_{2}$ ), and so, by (3), at most $n-1$ negative numbers $r_{i}$. Let $r_{i_{4}}$ be the smallest number among $r_{i}$ 's with $i$ of different parity than $i_{2}$, in particular $r_{i_{4}}>0$. Let $r_{i_{5}}$ be the smallest number among $r_{i}$ 's for $i$ of the same parity as $i_{2}$, in particular $r_{i_{5}} \leq-\frac{\theta-\rho}{2 n-2}$. So we have

$$
n r_{i_{4}}+(n-1) r_{i_{5}} \leq \sum\left\{r_{i}:(-1)^{i+i_{2}}=-1\right\}+\sum\left\{r_{i}:(-1)^{i+i_{2}}=1\right\}<\rho .
$$

Hence,

so

$$
n\left(r_{i_{4}}+r_{i_{5}}\right)<\rho+r_{i_{5}} \leq \rho-\frac{\theta-\rho}{2 n-2},
$$

$$
r_{i_{4}}+r_{i_{5}}<\frac{1}{n}\left(\rho-\frac{\theta-\rho}{2 n-2}\right)=\frac{(2 n-1) \rho-\theta}{n(2 n-2)},
$$

as required. 
Lemma 4.2. Let $n \geq 2$. Suppose that $\left(f_{\alpha}\right)_{\alpha<\omega_{1}}$ is a sequence of continuous rational simple functions on $K_{2 n}$ as in Theorem 3.8 and $\left(\mu_{\alpha}\right)_{\alpha<\omega_{1}}$ is a sequence of $(2 n-1)$-supported atomic Radon measures on $K_{2 n}$. Then either there are $\alpha<\beta<\omega_{1}$ such that

$$
\left|\int f_{\alpha} d \mu_{\beta}\right|>\frac{0.01}{2 n^{2}(2 n-2)}
$$

or there is $\alpha \in \omega_{1}$ such that

$$
\int f_{\alpha} d \mu_{\alpha}<0.99
$$

or there are $\alpha<\beta<\omega_{1}$ such that

$$
\int f_{\beta} d \mu_{\alpha}<-\frac{0.89}{2 n^{2}(2 n-2)} .
$$

Proof. By the separability of $C_{0} \equiv C\left(2^{\omega}\right)$ (see Definition 2.4), Lemma 2.6 and thinning out the sequence, we may assume that for all $\alpha<\omega_{1}$ we have

$$
f_{\alpha}=g+\sum_{1 \leq i \leq k} \sum_{1 \leq l \leq 2 n-1} q_{i, l} \chi_{A_{\xi_{\alpha}^{i}, l} \cap V_{s_{i}}}
$$

for some simple rational function $g \in C_{0}, F_{\alpha}=\left\{\xi_{\alpha}^{1}, \ldots, \xi_{\alpha}^{k}\right\} \subseteq \omega_{1}$, some $s_{i} \in 2^{m_{i}}, m_{i} \in N$ and some rationals $q_{i, l}, 1 \leq i \leq k$ and $1 \leq l \leq 2 n$, such that $s_{i}=r_{\xi_{\alpha}^{i}} \mid m_{i}$ and

$$
\sum_{1 \leq i \leq k}\left(\max _{1 \leq l \leq 2 n}\left|q_{i, l}\right|\right)\left|\mu_{\alpha}\right|\left(V_{s_{i}} \backslash R_{\xi_{\alpha}^{i}}\right) \leq \frac{0.01}{2 n^{2}(2 n-2)} .
$$

By thinning out the sequence (applying the $\Delta$-system lemma, see $[\mathrm{Ku}]$ ) and moving some identical parts to $g$ we may assume that $F_{\alpha}$ 's are pairwise disjoint and $g$ (no longer in $C_{0}$ ) is fixed. So, we will be allowed to use the following decompositions:

Claim 0. For each $\alpha, \beta<\omega_{1}$ we have

$$
\begin{aligned}
\int f_{\alpha} d \mu_{\beta}= & \int g d \mu_{\beta}+\sum_{1 \leq i \leq k} \sum_{1 \leq l \leq 2 n-1} q_{i, l} \mu_{\beta}\left(A_{\xi_{\alpha}^{i}, l} \cap R_{\xi_{\beta}^{i}} \cap V_{s_{i}}\right) \\
& +\sum_{1 \leq i \leq k} \sum_{1 \leq l \leq 2 n-1} q_{i, l} \mu_{\beta}\left(A_{\xi_{\alpha}^{i}, l} \cap V_{s_{i}} \backslash R_{\xi_{\beta}^{i}}\right) .
\end{aligned}
$$

Here, the last term is small by the above application of Lemma 2.6, the first term will be shown to be small by the claim below, and so the value of the integral will depend on the relation of the points from $R_{\xi_{\beta}, l}$ to the sets $A_{\xi_{\alpha}^{i}, l}$ which is "as we wish" on any uncountable set by Theorem 3.8 
Claim 1. Either (a) holds or for all but countably many $\alpha$ 's in $\omega_{1}$ we have

$$
\left|\int g d \mu_{\alpha}\right| \leq \frac{0.02}{2 n^{2}(2 n-2)} .
$$

Proof of the claim. If the inequality does not hold for uncountably many $\alpha$ 's, then by Theorem 3.8 we can find among them $\alpha<\beta<\omega_{1}$ such that $R_{\xi_{\beta}^{i}} \subseteq A_{\xi_{\alpha}^{i}, 2 n}$ for all $1 \leq i \leq k$. By Claim 0 we get

$$
\begin{aligned}
& \left|\int f_{\alpha} d \mu_{\beta}\right| \geq\left|\int g d \mu_{\beta}\right|-\sum_{1 \leq i \leq k} \sum_{1 \leq l \leq 2 n-1}\left|q_{i, l}\right|\left|\mu_{\beta}\left(A_{\xi_{\alpha}^{i}, l} \cap V_{s_{i}}\right)\right| \\
& \geq\left|\int g d \mu_{\beta}\right|-\sum_{1 \leq i \leq k}\left(\max _{1 \leq l \leq m}\left|q_{i, l}\right|\right)\left|\mu_{\beta}\right|\left(V_{s_{i}} \backslash R_{\xi_{\beta}^{i}}\right)>\frac{0.02-0.01}{2 n^{2}(2 n-2)}=\frac{0.01}{2 n^{2}(2 n-2)},
\end{aligned}
$$

proving (a) of the lemma and Claim 1.

Claim 2. Either (a) holds or for all but countably many $\alpha$ 's in $\omega_{1}$ we have, for each $1 \leq l_{0} \leq 2 n-1$,

$$
\left|\sum_{1 \leq i \leq k} q_{i, l_{0}} \mu_{\alpha}\left(R_{\xi_{\alpha}^{i}}\right)\right| \leq \frac{0.04}{2 n^{2}(2 n-2)} .
$$

Proof of the claim. Without loss of generality we may assume that the inequality of Claim 1 holds for all $\alpha<\beta<\omega_{1}$. Fix $l_{0}$ as above. Suppose that the inequality above does not hold for uncountably many $\alpha$ 's; then by Theorem 3.8 we obtain among them $\alpha<\beta$ such that for all $1 \leq i \leq k$,

$$
R_{\xi_{\beta}^{i}} \subseteq A_{\xi_{\alpha}, l_{0}}
$$

So by Claim 0 we have

$$
\begin{aligned}
\left|\int f_{\alpha} d \mu_{\beta}\right| \geq \mid \sum_{1 \leq i \leq k} \sum_{1 \leq l \leq 2 n-1} q_{i, l} \mu_{\beta}\left(R_{\xi_{\beta}^{i}}\right. & \left.\cap A_{\xi_{\alpha}^{i}, l} \cap V_{s_{i}}\right)|-| \int g d \mu_{\beta} \mid \\
& -\sum_{1 \leq i \leq k}\left(\max _{1 \leq l \leq 2 n-1}\left|q_{i, l}\right|\right)\left|\mu_{\beta}\right|\left(V_{s_{i}} \backslash R_{\xi_{\beta}^{i}}\right) \\
\geq\left|\sum_{1 \leq i \leq k} q_{i, l_{0}} \mu_{\beta}\left(R_{\xi_{\beta}^{i}}\right)\right|-\left|\int g d \mu_{\beta}\right| & -\sum_{1 \leq i \leq k}\left(\max _{1 \leq l \leq 2 n-1}\left|q_{i, l}\right|\right)\left|\mu_{\beta}\right|\left(V_{s_{i}} \backslash R_{\xi_{\beta}^{i}}\right) \\
& >\frac{0.04-0.02-0.01}{2 n^{2}(2 n-2)}=\frac{0.01}{2 n^{2}(2 n-2)},
\end{aligned}
$$

proving (a) and Claim 2.

Claim 3. Either (a) or (b) holds or there is $l_{0} \in\{1, \ldots, 2 n\}$ such that for uncountably many $\alpha$ 's in $\omega_{1}$ we have

$$
\sum_{1 \leq i \leq k} q_{i, l_{0}} \mu_{\alpha}\left(\left\{\left(x_{\xi_{\alpha}^{i}}, l_{0}\right)\right\}\right)>\frac{0.96}{2 n} .
$$


Proof of the claim. Assume that (a) does not hold, i.e., the inequalities of Claims 1 and 2 hold for all $\alpha<\omega_{1}$. Now, suppose also that the inequality above does not hold for any $l_{0} \in\{1, \ldots, 2 n\}$. By Claim 0 for $\alpha=\beta$ we have

$$
\begin{aligned}
\int f_{\alpha} d \mu_{\alpha} \leq \sum_{1 \leq i \leq k} \sum_{1 \leq l \leq 2 n-1} q_{i, l} \mu_{\alpha}\left(\left\{\left(x_{\xi_{\alpha}^{i}}, l\right)\right\}\right) & \\
& \quad-\frac{0.02}{2 n^{2}(2 n-2)}-\frac{0.01}{2 n^{2}(2 n-2)} \leq \frac{(2 n-1) 0.96}{2 n}-0.03<0.99,
\end{aligned}
$$

that is, we obtain (b), which concludes the proof of Claim 3.

To finish the proof of the lemma, we assume that (a) and (b) fail, i.e., the inequalities of all the above claims hold, and we need to get (c). Fix $\alpha<\omega_{1}$; we will apply Lemma 4.1 for

$$
r_{l, \alpha}=\sum_{1 \leq i \leq k} q_{i, l_{0}} \mu_{\alpha}\left(\left\{\left(x_{\xi_{\alpha}^{i}}, l\right)\right\}\right)
$$

and $l \in\{1, \ldots, 2 n\}$. Since the supports of the measures $\mu_{\alpha}$ have at most $2 n-1$ elements, one of $r_{l, \alpha}$ 's must be zero. By Claim 3 we have $r_{l_{0}, \alpha}>\theta=$ $0.96 /(2 n)$ and by Claim $2, \sum_{1 \leq l \leq 2 n} r_{l, \alpha}<\rho=0.04 /(2 n)^{2}$. So by Lemma 4.1 we find $1 \leq l_{1, \alpha}, l_{2, \alpha} \leq 2 n$ of different parities such that

$$
\begin{aligned}
\sum_{1 \leq i \leq k} q_{i, l_{0}} \mu_{\alpha}\left(\left\{\left(x_{\xi_{\alpha}^{i}}, l_{1, \alpha}\right),\left(x_{\xi_{\alpha}^{i}}, l_{2, \alpha}\right)\right\}\right)<\frac{2 n \rho-\theta}{n(2 n-2)} & \\
= & \frac{2 n\left(0.04 /(2 n)^{2}\right)-0.96 /(2 n)}{n(2 n-2)}=-\frac{0.92}{2 n^{2}(2 n-2)} .
\end{aligned}
$$

We may assume that $l_{1, \alpha}=l_{1}$ and $l_{2, \alpha}=l_{2}$ for all $\alpha<\omega_{1}$. Note that by Theorem 3.8 we can find $\alpha<\beta<\omega_{1}$ such that

$$
\left\{\left(x_{\xi_{\alpha}^{i}}, l_{1}\right),\left(x_{\xi_{\alpha}^{i}}, l_{2}\right)\right\} \subseteq A_{\xi_{\beta}^{i}, l_{0}}
$$

and

$$
R_{\xi_{\alpha}^{i}} \backslash\left\{\left(x_{\xi_{\alpha}^{i}}, l_{1}\right),\left(x_{\xi_{\alpha}^{i}}, l_{2}\right)\right\} \subseteq A_{\xi_{\beta}^{i}, 2 n}
$$

for all $1 \leq i \leq k$. Together with Claim 0 with $\alpha$ and $\beta$ switched, this implies that

$$
\begin{aligned}
\int f_{\beta} d \mu_{\alpha} \leq & \sum_{1 \leq i \leq k} q_{i, l_{0}} \mu_{\alpha}\left(\left\{\left(x_{\xi_{\alpha}^{i}}, l_{1}\right),\left(x_{\xi_{\alpha}^{i}}, l_{2}\right)\right\}\right) \\
& +\sum_{1 \leq i \leq k}\left(\max _{1 \leq l \leq 2 n-1}\left|q_{i, l}\right|\right)\left|\mu_{\alpha}\right|\left(V_{s_{i}} \backslash R_{\xi_{\alpha}^{i}}\right)+\left|\int g d \mu_{\alpha}\right| \\
\leq & \frac{-0.92+0+0.01+0.02}{(2 n)^{2}(2 n-2)}=-\frac{0.89}{(2 n)^{2}(2 n-2)},
\end{aligned}
$$

which completes the proof of the lemma. 
TheOREM 4.3. Let $n \geq 2$. If $K_{2 n}$ is an unordered $2 n$-split Cantor set as in Theorem 3.8, then there are no uncountable semibiorthogonal sequences in $C\left(K_{2 n}\right)$ whose functionals are $(2 n-1)$-supported but there is an uncountable biorthogonal system whose functionals are $2 n$-supported.

Proof. Suppose $\left(f_{\alpha}, \mu_{\alpha}\right)_{\alpha<\omega_{1}} \subseteq C\left(K_{2 n}\right) \times M\left(K_{2 n}\right)$ is a sequence whose functionals are $2 n-1$-supported and that $\int f_{\alpha} d \mu_{\beta}=0$ for all $\alpha<\beta<\omega_{1}$ as well as $\int f_{\alpha} d \mu_{\alpha}=1$ for all $\alpha<\omega_{1}$.

We may assume without loss of generality that $\left\|\mu_{\alpha}\right\| \leq M$ for some positive $M$. By the Stone-Weierstrass theorem we can choose $f_{\alpha}^{\prime} \in C(K)$ which is a rational simple function and

$$
\left\|f_{\alpha}^{\prime}-f_{\alpha}\right\|<\frac{0.01}{2 M n^{2}(2 n-2)} .
$$

This means that (a) and (b) of Lemma 14 do not hold for $f_{\alpha}^{\prime}$ 's in place of $f_{\alpha}$ 's, i.e. (c) holds, which implies that $\left(f_{\alpha}, \mu_{\alpha}\right)_{\alpha<\omega_{1}}$ is not semibiorthogonal.

TheOREM 4.4. If $K_{2 n}$ is an unordered $2 n$-split Cantor set as in Theorem 3.8, then $h d\left(K_{2 n}^{n}\right)=\omega$.

Proof. We will be using the well-known fact that a regular space is hereditarily separable if and only if it has no uncountable left-separated sequence (see Theorem 3.1 of [16]).

Suppose $\left(y_{\alpha}\right)_{\alpha<\omega_{1}}$ is a left-separated sequence in $K_{2 n}^{n}$ of cardinality $\aleph_{1}$. Hence, for each $\alpha<\omega_{1}, y_{\alpha}=\left(y_{\alpha}^{1}, \ldots, y_{\alpha}^{n}\right)$, where each $y_{\alpha}^{m} \in K_{2 n}$ and, by the definition of a left-separated sequence, for each $\alpha<\omega_{1}$ and each $m \in[N]$, there is an open basic neighbourhood $U_{\alpha}^{m}$ of $y_{\alpha}^{m}$ such that

$$
\forall \alpha<\omega_{1} \forall m \in[n] \quad y_{\alpha}^{m} \in U_{\alpha}^{m}
$$

and

$$
\forall \alpha<\beta<\omega_{1} \exists m \in[n] \quad y_{\alpha}^{m} \notin U_{\beta}^{m} .
$$

We may assume without loss of generality that

$$
\left\{m \in[n]: y_{\alpha}^{m} \in 2^{\omega} \backslash\left\{x_{\xi}: \xi<\omega_{1}\right\}\right\}=\left\{m \in[n]: y_{\beta}^{m} \in 2^{\omega} \backslash\left\{x_{\xi}: \xi<\omega_{1}\right\}\right\}
$$

for every $\alpha<\beta<\omega_{1}$ and let us call this set $I$.

For each $m \in[n] \backslash I$, let $\xi_{\alpha}^{m}$ be a countable ordinal and $j_{\alpha}^{m}$ be an element of $[n]$ such that $y_{\alpha}^{m}=\left(x_{\xi_{\alpha}^{m}}, j_{\alpha}^{m}\right)$.

Now, for each $m \in[n]$, let $s_{\alpha}^{m} \in 2^{<\omega}$ be such that

$$
U_{\alpha}^{m}= \begin{cases}V_{s_{\alpha}^{m}} & \text { if } m \in I, \\ V_{s_{\alpha}^{m}} \cap A_{\xi_{\alpha}^{m}, j_{\alpha}^{m}} & \text { if } m \notin I .\end{cases}
$$

Put $E_{\alpha}=\left\{\xi_{\alpha}^{m}: m \in[n] \backslash I\right\}$.

Without loss of generality, we may assume that:

- there is $j_{m} \in[n]$ such that $j_{\alpha}^{m}=j_{m}$ for all $\alpha<\omega_{1}$; 
- there is $s_{m} \in 2^{<\omega}$ such that $s_{\alpha}^{m}=s_{m}$ for all $\alpha<\omega_{1}$ (this already guarantees that each $\left.y_{\alpha}^{m} \in V_{s_{m}}\right)$;

- for all $m \in[n] \backslash I$, either

$$
\forall \alpha<\beta<\omega_{1} \quad \xi_{\alpha}^{m}=\xi_{\beta}^{m},
$$

or

$$
\forall \alpha<\beta<\omega_{1} \quad \xi_{\alpha}^{m}<\xi_{\beta}^{m} .
$$

- $\left(E_{\alpha}\right)_{\alpha<\omega_{1}}$ is a $\Delta$-system with root $\Delta$ such that for every $\alpha<\beta<\omega_{1}$, $\Delta<E_{\alpha} \backslash \Delta<E_{\beta} \backslash \Delta$ and $\left|E_{\alpha}\right|=\left|E_{\beta}\right|$.

If $E_{\alpha} \backslash \Delta=\emptyset$, the left-separated sequence in $K_{2 n}^{n}$ would lead to a leftseparated sequence in a finite power of $2^{\omega}$, which is not possible since $2^{\omega}$ is hereditarily separable in all finite powers. Therefore, each $E_{\alpha} \backslash \Delta \neq \emptyset$ and they are pairwise disjoint.

For each $\alpha<\omega_{1}$, enumerate $E_{\alpha} \backslash \Delta=\left\{\eta_{\alpha}^{1}<\cdots<\eta_{\alpha}^{k}\right\}$. We may assume that $\xi_{\alpha}^{m}=\eta_{\alpha}^{i}$ if and only if $\xi_{\beta}^{m}=\eta_{\beta}^{i}$.

Claim. For each $1 \leq i \leq k$, one can find $I_{i} \subseteq[2 n]$ of cardinality $N$ and a bijection $\sigma_{i}: I_{i} \rightarrow[2 n] \backslash I_{i}$ such that $\sigma_{i}(l)$ and $l$ have opposite parity and

$$
\left\{j \in[2 n]: \exists m \in[n] \text { such that } j=j_{m} \text { and } \xi_{\alpha}^{m}=\eta_{\alpha}^{i}\right\} \subseteq I_{i} .
$$

Proof of the claim. The claim follows easily from the fact that the set

$$
\left\{j \in[2 n]: \exists m \in[n] \text { such that } j=j_{m} \text { and } \xi_{\alpha}^{m}=\eta_{\alpha}^{i}\right\}
$$

has cardinality at most $n$ so that we can find $I_{i}$ containing it, and that whenever we have a partition of $[2 n]$ into two sets $A$ and $B$, both of size $n$, then $A$ has as many odds as $B$ has evens, and vice versa.

Now, let $\epsilon:[k] \times[2 n] \rightarrow[2 n]$ be defined by

$$
\epsilon(i, l)= \begin{cases}l & \text { if } l \in I_{i}, \\ \sigma_{i}^{-1}(l) & \text { if } l \in[2 n] \backslash I_{i} .\end{cases}
$$

Notice that for each $i \in[k], l \in I_{i}$ and $j \in[2 n], \epsilon(i, l)=j$ if and only if $\epsilon(i, \sigma(l))=j$. Since $\sigma(l)$ and $l$ have opposite parities, we see that $\epsilon$ has the desired property, that is,

$|\{l \in\{1,3,5, \ldots, 2 n-1\}: \epsilon(i, l)=j\}|=|\{l \in\{2,4,6, \ldots, 2 n\}: \epsilon(i, l)=j\}|$.

By Theorem 3.8, there are $\alpha<\beta$ such that for all $i \in[k]$,

$$
\left(x_{\eta_{\alpha}^{i}}, l\right) \in A_{\eta_{\beta}^{i}, \epsilon(i, l)}
$$

Fix $m \in[n]$ and let us prove that $y_{\alpha}^{m} \in U_{\beta}^{m}$, contradicting the assumption. If $m \notin I$, then $y_{\alpha}^{m} \in V_{s_{m}}=U_{\beta}^{m}$. If $m \in I$ and $\xi_{\alpha}^{m} \in \Delta$, then $\xi_{\alpha}^{m}=\xi_{\beta}^{m} \in U_{\beta}^{m}$. Finally, if $m \in I$ and $\xi_{\alpha}^{m} \notin \Delta$, then there is $i \in[k]$ such that $\xi_{\alpha}^{m}=\eta_{\alpha}^{i}$ 
and $\xi_{\beta}^{m}=\eta_{\beta}^{i}$. In this case we have $j_{m} \in I_{i}$, and so $\epsilon\left(i, j_{m}\right)=j_{m}$, which guarantees that

$$
y_{\alpha}^{m}=\left(x_{\xi_{\alpha}^{m}}, j_{m}\right)=\left(x_{\eta_{\alpha}^{i}}, j_{m}\right) \in A_{\eta_{\beta}^{i}, j_{m}}=A_{\xi_{\beta}^{m}, j_{m}} .
$$

Since also $y_{\alpha}^{m} \in V_{s_{m}}$, we get $y_{\alpha}^{m} \in U_{\beta}^{m}$, which concludes the proof.

Acknowledgements. We would like to thank Szymon Głąb for noting a gap in the previous proof of Lemma 4.1 and for correcting it.

The first author was supported by FAPESP fellowship (2007/08213-2), which is part of Thematic Project FAPESP (2006/02378-7). Part of the research was done at the Technical University of Eódź where the first author was partially supported by Polish Ministry of Science and Higher Education research grant N N201 386234.

The second author was partially supported by Polish Ministry of Science and Higher Education research grant N N201 386234. Part of the research was done at the State University of Campinas UNICAMP where the second author was partially supported by the Department of Mathematics.

\section{References}

[1] M. Bell, J. Ginsburg, and S. Todorčević, Countable spread of $\exp Y$ and $\lambda Y$, Topology Appl. 14 (1982), 1-12.

[2] J. M. Borwein and J. D. Vanderwerff, Banach spaces that admit support sets, Proc. Amer. Math. Soc. 124 (1996), 751-755.

[3] C. Brech and P. Koszmider, Thin-very tall compact scattered spaces which are hereditarily separable, Trans. Amer. Math. Soc. 363 (2011), 501-519.

[4] M. Džamonja and I. Juhász, CH, a problem of Rolewicz and bidiscrete systems, Topology Appl., to appear.

[5] R. Engelking, General Topology, 2nd ed., Sigma Ser. Pure Math. 6, Heldermann, Berlin, 1989.

[6] M. Fabian, P. Habala, P. Hájek, V. Montesinos Santalucía, J. Pelant, and V. Zizler, Functional Analysis and Infinite-Dimensional Geometry, CMS Books Math./Ouvrages Math. SMC 8, Springer, New York, 2001.

[7] C. Finet and G. Godefroy, Biorthogonal systems and big quotient spaces, in: Banach Space Theory (Iowa City, IA, 1987), Contemp. Math. 85, Amer. Math. Soc., Providence, RI, 1989, 87-110.

[8] A. S. Granero, M. Jiménez Sevilla, and J. P. Moreno, Convex sets in Banach spaces and a problem of Rolewicz, Studia Math. 129 (1998), 19-29.

[9] P. Hájek, V. Montesinos Santalucía, J. Vanderwerff, and V. Zizler, Biorthogonal Systems in Banach Spaces, CMS Books Math./Ouvrages Math. SMC 26, Springer, New York, 2008.

[10] M. Katětov, Complete normality of Cartesian products, Fund. Math. 35 (1948), 271-274.

[11] P. Koszmider, On a problem of Rolewicz about Banach spaces that admit support sets, J. Funct. Anal. 257 (2009), 2723-2741. 
[12] K. Kunen, Set Theory. An Introduction to Independence Proofs, Stud. Logic Found. Math. 102, North-Holland, Amsterdam, 1980.

[13] A. J. Lazar, Points of support for closed convex sets, Illinois J. Math. 25 (1981), 302-305.

[14] J. D. Monk, Cardinal Functions on Boolean Algebras, Lectures in Math. ETH Zürich, Birkhäuser, Basel, 1990.

[15] S. Negrepontis, The Stone space of the saturated Boolean algebras, Trans. Amer. Math. Soc. 141 (1969), 515-527.

[16] J. Roitman, Basic $S$ and L, in: Handbook of Set-Theoretic Topology, North-Holland, Amsterdam, 1984, 295-326.

[17] S. Rolewicz, On convex sets containing only points of support, Comment. Math. Special Issue 1 (1978), 279-281,

[18] S. Shelah, On uncountable Boolean algebras with no uncountable pairwise comparable or incomparable sets of elements, Notre Dame J. Formal Logic 22 (1981), 301-308.

[19] -, Uncountable constructions for B.A., e.c. groups and Banach spaces, Israel J. Math. 51 (1985), 273-297.

[20] I. Singer, Bases in Banach Spaces. I, Grundlehren Math. Wiss. 154, Springer, New York, 1970.

[21] —, Bases in Banach Spaces. II, Editura Academiei, Bucureşti, 1981.

[22] Z. Szentmiklóssy, $S$-spaces and L-spaces under Martin's axiom, in: Topology, Vol. II (Budapest, 1978), Colloq. Math. Soc. János Bolyai 23, North-Holland, Amsterdam, 1980, 1139-1145.

[23] S. Todorcevic, Irredundant sets in Boolean algebras, Trans. Amer. Math. Soc. 339 (1993), 35-44.

[24] -, Biorthogonal systems and quotient spaces via Baire category methods, Math. Ann. 335 (2006), 687-715.

Christina Brech

Instituto de Matemática, Estatística

e Computação Científica

Universidade Estadual de Campinas

Rua Sérgio Buarque de Holanda 651

13083-859, Campinas, Brazil

Current address:

Instytut Matematyki Politechniki Łódzkiej

Wólczańska 215

90-924 Łódź, Poland

E-mail: christina.brech@gmail.com
Piotr Koszmider

Instytut Matematyki Politechniki Łódzkiej

Wólczańska 215

90-924 Łódź, Poland

E-mail: pkoszmider.politechnika@gmail.com

Current address: Institute of Mathematics Polish Academy of Sciences

Sniadeckich 8

P.O. Box 21

00-956 Warszawa, Poland

E-mail: piotr.math@gmail.com

Received 19 May 2010;

in revised form 3 March 2011 